Portland State University

PDXScholar

$5-23-1975$

\title{
Alternative Methods for the Analysis of Curves
}

Randi Christine Martin

Portland State University

Follow this and additional works at: https://pdxscholar.library.pdx.edu/open_access_etds

Part of the Psychology Commons

Let us know how access to this document benefits you.

\section{Recommended Citation}

Martin, Randi Christine, "Alternative Methods for the Analysis of Curves" (1975). Dissertations and Theses. Paper 2379.

https://doi.org/10.15760/etd.2375

This Thesis is brought to you for free and open access. It has been accepted for inclusion in Dissertations and Theses by an authorized administrator of PDXScholar. Please contact us if we can make this document more accessible: pdxscholar@pdx.edu. 
AN ABSTRACT OF THE THESIS OF Randi Christine Jertin for the Master of Science in Psychology presented May 23, 1975.

Title: Alternative Methods for the Analysis of Curves. APPROVED BY MEMEBERS OF THE THESIS COMMITTEE:
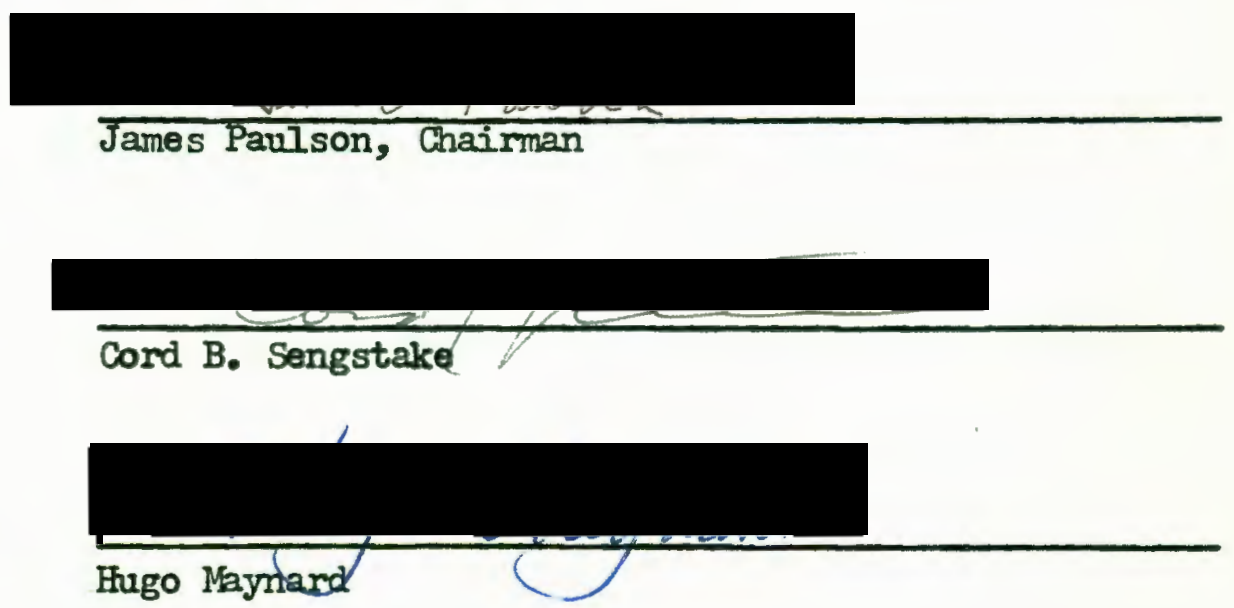

The interpretation of data from psychological experiments often Involves the analysis and comparison of curves, like the electrocardiogram, spectral sensitivity, or dose-response curves. Edisting statistical techniques are often inadequate for making this type of analysis. This thesis presents alternative methods for handling the data comprising curves and discusses the advantages of these techniques against those of existing methods.

Four types of analysis are discussed:

1) finding a confidence band around one curve,

2) finding a confidence band around the difference between two curves, or making an overall comparison between two curves, 
3) decomposing a curve into its components,

4) making a component by component comparison of two curves.

The alternative methods presented for the first two types of analysis involve finding simultaneous confidence intervals on the points making up a curve or on the difference between two curves. The first type of analysis, that of finding a confidence band around one curve, is not presently used in psychological studies. The second type of analysis, that of making an overall comparison betwoen two curves, is presently accomplished by visual inspection, analysis of variance, or Hotelling's $\mathrm{T}^{2}$.

For the third and fourth types of analysis the use of a set of orthogonal functions known as Haar functions is presented as an alternative to the use of orthogonal polynomials.

For each type of analysis, two cases are presented, each of which makes different assumptions about the shape of the covariance matrix. Case I assumes that the covariance matrix is unknown, while case II assumes that the covariance matrix is known except for a scalar. To illustrate the altemative techniques for case I, data from an actual experimeit on the effect of $\Delta^{\prime}-T H C$ (tetrahydrocannabinol) on the p-wave of the electrocardiogram is used. To illustrate case II, simulated data is used involving a dose-response curve generated by testing independent graups on a memory task at four levels of a synthetic analogue of $\Delta^{9}-$ THC. This is compared to a dose-response curve testing independent groups on the same task at the same dosage levels of $\Delta^{9}$-THC.

As was the case for the examples, the p-wave and the dose-response curves, the alternative techniques provide adequate means 
for the analysis and comparison of curves. In some cases, as in the use of Haar functions for the decomposition of the p-save, the alternative techniques provide advantages over existing methods. It is hoped that the new techniques will be incorporated into the analysis of psychological date. 
ALTERNATIVE METHODS FOR THE ANALYSIS OF CURVES

by
RANDI CHRISTINE MARTIN

A thesis submitted in partial fulfillment of the requirements for the degree of

\section{MASTER OF SCIENGEF \\ in \\ PSYCHOLOGY}

Portland State University

1975 
TO THE OFFICE OF GRADUATE SIUDIES AND RESFARCH:

The members of the Committee approve the thesis of

Randi Christine Martin presented May 23, 1975.

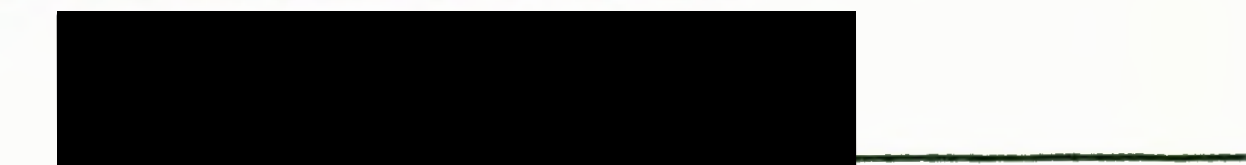

James Paulson, Chairman
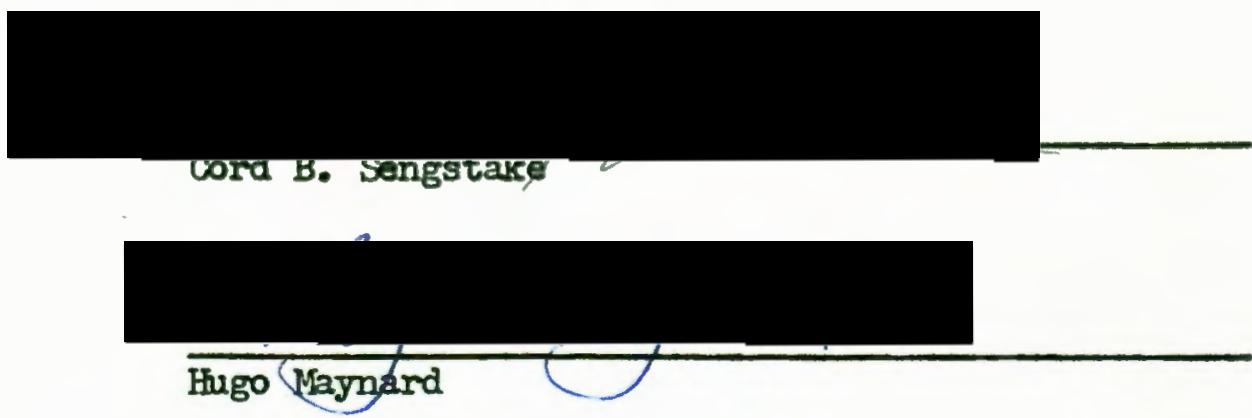

APPBOVED:

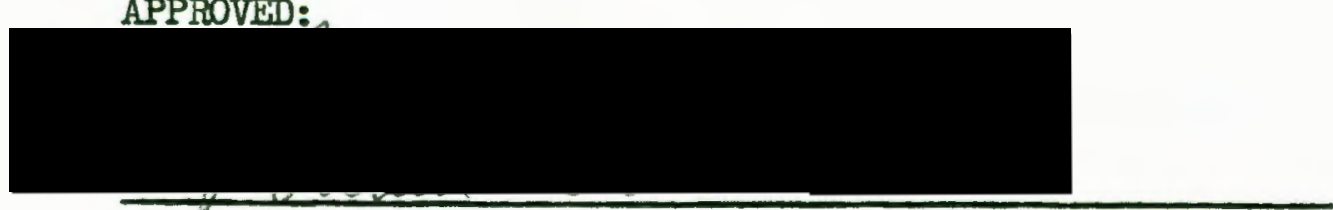

Ronáld E. Smith, Head, Department of Psychology

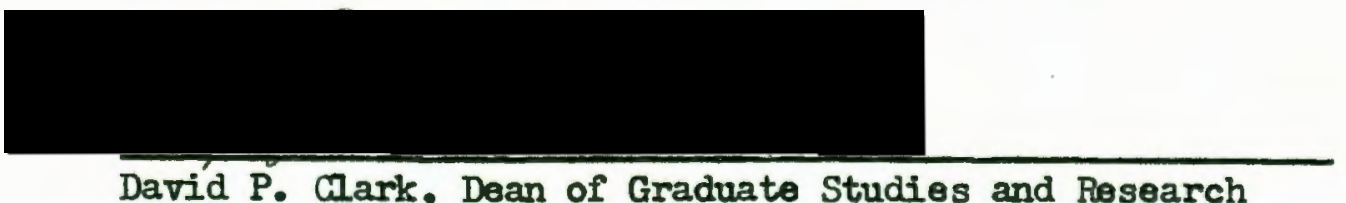

David P. Gark, Dean of Graduate Studies and Research

July 23, 1975 
I would like to express my thanks to the members of my committee for the assistance they have given me in the preparation of this thesis. Through the several months of working on this thesis, Dr. James Paulson has given many helpful suggestions and criticisms as to the statistical content and organization of ideas. Dr. Cord Sengstake and $\mathrm{Dr}$. Hugo Maynard were helpful in suggesting changes which would make the material more accessible to the general body of psychologists who lack an extensive mathematical background. I would also like to thank Dr. Sengstake for the use of his data on the electrocardiogram and for his assistance in the use of the PDP-11 computer to transform this data into usable form. 
TABLE OF CONTENTS

PAGE

ACKNOWIETGMENTS ....................... 1....

IIST OF TARLES. ...................... vi

IIST OF FIGURES ....................... viI

\section{SECTION}

I. INTRODUCTION .................. 1

II. FINDING A CONFIDENCE BAND AROUND ONE CJRVE .... 4

Case I: Covariance Matrix Unknown ....... 5

Case II: Covariance Matrix Known

Except for a Scalar. ... 7

III. FINDING A CONFIDENCE BAND FOR THE

DIFFERENCE BETWEEN TWO CURVES. ..... 出

Case I: Covariance Matrix Unknown ..... 18

Case II: Covariance Matrix Known

Except for a scalar. ... 18

IV. DECOMPOSITION OF A CURVE INTO ITS COMPONENTS . - - 25

Orthogonal Polynomials ......... 29

Haar Functions .......... 33

Case I: Covariance Matrix Unknown ...... 42

Case II: Covariance Matrix Known

Except for a Scalar. ... 49

V. CONPONENT BI COMPONENT COMPARISON OF TWO CURVES. . 50

Case I: Covariance Matrix Unknown ... . . 57

Case II: Covariance Matrix Inown

Except for a Scalar. ... 61 
PAGE

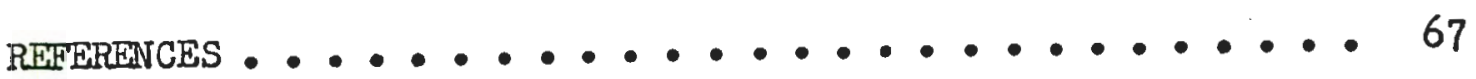

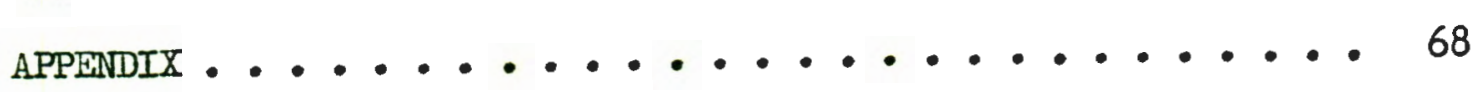


LIST OF TABLES

TABTE

PAGE

I Mean, Variance, and Confldence Interval for Average

P-wave. .....................

II Performance of Independent Groups on Memory Task at Four

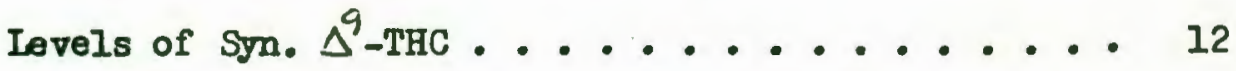

III Average Score and Confidence Interval around Each Point

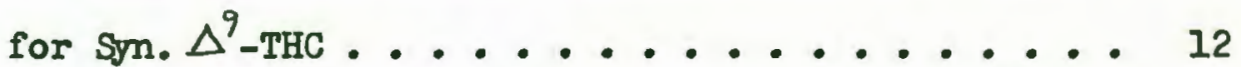

IV Confidence Band about Difference between Average P-waves

Before and After Administration of $\Delta^{9}$ THC ..... 19

$\nabla$ Performance of Independent Groups on Memory Task at Four

Ievels of $\Delta^{9}$-THC. ............. 23

VI Confidence Band around Difference between Average Sym.

$\Delta^{9}$-THC Scores and Average $\Delta^{9}$ THC scores ...... 23

VII Haar Coefficients for a Curve Comprised of Four, Eight

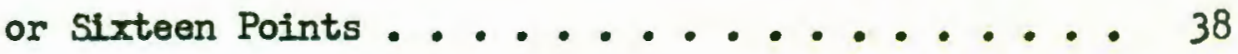

VII Haar Coefficients for a Curve Comprised of Seven Points . 40

IX Sum of Squares for Haar Components of Curve in Flgure 9 - 41

$X$ Haar Components of P-wave Before Administration of $\triangle^{9}$-THC 46

XI Haar Components of Syn. $\triangle^{9}$ THC Performance Curve. . . 52

XII Polynomial Components of Syn. $\Delta^{9}$-THC Performance Curve. . 52

XIII Comparison of Haar Components of P-waves Before and After

Administration of $\triangle \Delta^{9}$ THC. .......... 58

XIV Comparison of Haar Components of Dose-Response Curves

of Syn. $\triangle^{9}$ THC and $\triangle^{9}$ THC ............ 62 
IIST OF FIGURES

FIGURE

PAGE

1 Confidence band around average p-wave ......... 9

2 Confidence band around performance scores at four dosage

levels of a synthetic analogue of $\Delta^{9}-T H C . . . .13$

3 Average p-wave before and after administration $\triangle 9-T H C$. 20

4 Confidence band about difference between average p-waves shown in figure 3............... 21

5a Curve 1 shows average performance score at four dosage levels of syn. $\Delta^{9}$-THC. Qurve 2 shows the same for

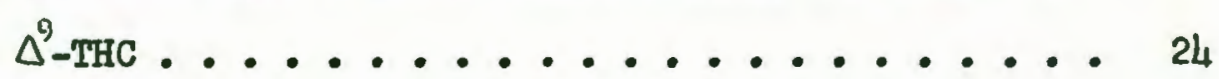

$5 \mathrm{~b}$ Confidence band about the difference between the two

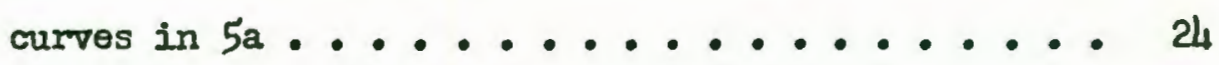

6 Curve $6 a$ shows a difference in level between the first half and the second half. Curre $6 b$ shows a Iinear

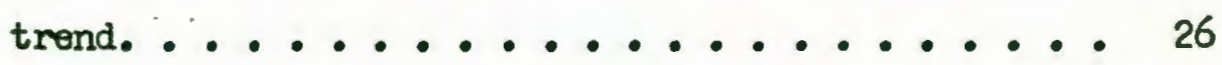

7 Curves show the first four polynomial trends. . . . . 30

8 Curves show variations in polynomial trends ....... 32

9 The first, third and fifth polynomial trends are significant in this simple, but non-symetric curve... 32

10 The first four Haar functions ............ 34

11 Curves illustrating significant or non-signiflcant components of the second Haar function....... 35

12 Curves illustrating significant components of the third

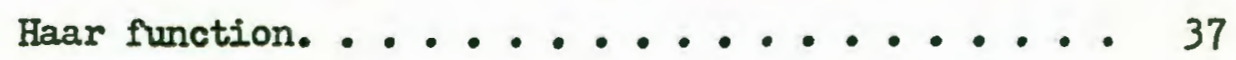

13 Average p-wave before administration of $\triangle^{c}-T H C$ and recon- 
viii

PAGE

struction of the curve from the significant Haar com-

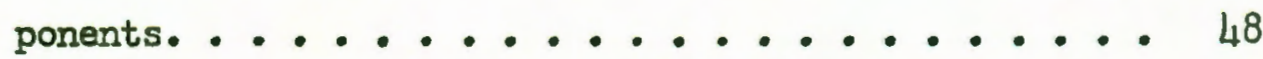

I4 Gurve of average performance scores under syn. $\Delta^{9}-$ THC and reconstruction of the curve from the significant Haar

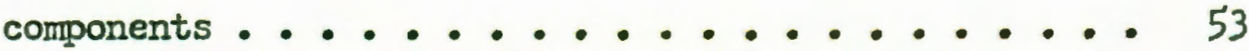




\section{INTRODUCTION}

Many problems within psychology deal with the relationship of a dependent variable to an independent variable which varies quantitatively along some continuum. For example, a learning curve shows the relationship between number of correct responses and number of trials. Another exarmle is a waveform such as an electrocardiogram, thought of in its digital representation, which shows voltage over time. A third example would be spectral sensitivity measured over various wavelengths of light. Typically this relationship of independent variable to dependent variable is charted as some form of curve and this curve compared to another curve obtained under another experimental condition, or to one predicted by some theoretical relation between the two variables.

The previously mentioned examples fould most likely involve repeated measures on the same subject, that is, the same subject tested at each level of the independent variable. However, experiments involving independent groups for the different levels could also give rise to data in the form of curves. An example would be a study involving performance on a certain task after administration of differing doses of a drug with independent groups at each dosage level. These results could be graphed as a dose-response curve.

Although this type of data is common in psychology, the excisting statistical techniques for dealing with curves are often inadequate and for some types of data non-existent. The intent of this thesis will be 
to propose techniques for dealing with the analysis of curves. A first objective will be to review existing methods of curve analysis pointing out their assumptions and types of problems to which they are most applicable. Secondly, I will propose alternative techniques which for some kinds of problems will provide advantages over existing methods. Four kinàs of analysis are typically made when considering the data comprising curves:

1) finding a confidence interval around each of the points making up a curve, or equivalently, finding a confidence band around the entire curve, 2) finding an overall difference between two or more curves, or the confidence band for the difference between two or more curves, 3) decomposing a curve into its components, 4) making a component by component comparison of two or more curves. These four kinds of analysis will be discussed in sections II through $\nabla$ of this paper.

Before beginning the discussion of techniques some explanation of the notation used will be necessary:

lower case letters will represent scalars: a, b, $\pi, \quad,, \alpha$ lower case letters which are underlined will represent vectors: $\underline{x}, \underline{\mathbb{y}}, \underline{\mu}$ capital letters will represent matrices: $S, X, \Sigma$

The most commonly used vectors will be $\overline{\mathrm{Y}}$ and $\mu \cdot \overline{\mathrm{y}}$ is the sample mean vector which is a set of measurements showing the average value of the dependent variable at each level of the independent variable. For example if the following values of the dependent variable $y$ were found for the following values of $x\left(x=x_{1}, x_{2}, x_{3}, x_{4}\right)$ : 


\begin{tabular}{ccccc} 
& \multicolumn{6}{c}{$x$} \\
\cline { 2 - 5 }$y$ & $x_{1}$ & $x_{2}$ & $x_{3}$ & $x_{4}$ \\
\cline { 2 - 5 } & 98 & 110 & 120 & 126 \\
average & 100 & 112 & 113 & 130 \\
& 102 & 107 & 115 & 110 \\
\hline & 100 & 109.6 & 116 & 132
\end{tabular}

$\bar{y}$ would equal $(100,109.6,116,132)$. The elements of $\bar{y}$ will be labelled $\bar{y}_{i}$ where $\overline{\underline{z}}=\left(\bar{y}_{1}, \bar{y}_{2}, \bar{y}_{3}, \bar{y}_{4}, \ldots \ldots, \bar{y}_{p}\right)$ given $p$ levels of the independent variable. In the previous example:

$$
\bar{y}_{1}=100, \quad \bar{y}_{2}=109.6, \quad \bar{y}_{3}=116, \quad \bar{y}_{4}=132 .
$$

$\mu$ is the population mean vector and is not a random vector. $\bar{Z}$ is an estimate of $\mu$.

The most commonly used matrics will be $\Sigma$ and S. $\Sigma$ is the variancecovariance matrix of $\mu$ and $S$ is the estimate of this matrix. In all cases $\overline{\mathrm{Z}}$ will be assumed to have a maltivariate normal distribution with mean $\mu$ and covariance $\Sigma$.

Also, the mathematical symbols $\epsilon$ and $\forall$ will be used. $\epsilon$ means 'is contained in' and $\forall$ means 'for every.'

Vector multiplication and matrix multiplication will be used to simplify notation. The appendix gives an explanation of how to perform vector and matrix maltiplication. 
II. FINDING A CONFIDENCE BAND AROUND ONE CURVE

Returning to the types of analysis made when dealing with curves, the first to be considered will be that of finding a confidence band around a curve. This is sinilar to the univariate problem of finding a confidence interval around the mean of a sample. In the univariate case, one finds the mean and uses tables of the t-distribution to find the interval:

$$
\mu \in z+t_{n-1}^{\alpha / 2} \frac{s}{\sqrt{n-1}}
$$

In the case of curves, one wishes to arrive at a confidence band for the entire curve. For example, suppose that an experimenter has fifty observations of an electrocardiogram and wishes to find a confidence band for the average of these fifty. A natural inclination is to calculate the interval described above for each of the points making up the curve using an $\alpha$ of .05 for each point This is incorrect, however. Taking an $\alpha$ of .05 means that there is a probability of .05 that the confidence interval will not contain the true mean, $\mu_{i}$, for that point. The probability that any part of the curve could lie outside the confidence band for the entire curve will be greater than .05 because we are concermed with the joint probability that one or more confidence intervals will not contain the true mean for that point while the remaining confidence intervals could cover their $\mu_{i}$. The value of the joint probability will depend on the degree to which the points are correlated. Only if the points are all perfectly correlated will the $\alpha$ level for the 
entire curve be as small as that for each point. If the points are all independent, the probability that the confidence band will cover all the points would be the product of the probabilities for the individual confidence intervals. For a curve comprised of four points with each confidence interval having a probability of .95 of covering the true mean, the joint probability for incependent points would be

$$
(.95) \times(.95) \times(.95) \times(.95)=.815
$$

not an acceptable confidence level.

Since in actual data the points will most likely not be perfectly correlated, the simultaneous probability will be less than $1-\alpha$. An alternative technique should provide confidence intervals for each of the points all of which hold similtaneously with probability $1-\alpha$. The simultaneous multivariate techniques to be described in this paper were originally developed by Roy and Bose (1953) and are summarized in Miller's Simultaneous Statistical Inference (1966).

Miller presents two cases in the discussion of finding a confidence interval around a curve. One must determine if his data fit the assumptions of the first case or the second. In the first case, the covariance matrix is completely unknown. Most data encountered in the comparison of curve will fall under this case due to lack of knowledge about the shape of the covariance matrix. The second case assumes that the covariance matrix is known except for a scalar.

CASE I: COVARIANGE MATRIX UNKNONN

Either of tivo expressions may be used to determine confidence intervals about the co-ordinate means: 
(I) $\quad \mu_{i} \in \bar{y}_{i} \pm t_{n-I}^{\alpha / 2 n}\left(\frac{s_{i}^{2}}{n}\right)^{1 / 2}$

$i=1, \ldots, p$

where $s_{i}^{2}=\frac{I}{n-1} \sum_{j=1}^{n}\left(y_{i j}-\bar{y}_{i}\right)^{2}$, or

$$
u \in{\overline{y_{i}}}_{i} \pm\left(p F_{p_{2}, n-p}^{\alpha}\right)^{1 / 2}\left(\frac{n-1}{n-p}\right)^{1 / 2}\left(\frac{s_{i}^{2}}{n}\right)^{1 / 2} \quad i=1, \ldots, p .
$$

Expression ( 1 ) is based on the Bonferroni inequality. If an overall a level of significance is desired, using an $\alpha / 2 p$ confidence level for each point (where $p$ is the number of points) will, according to the Bonferroni inequality, assure that the p statements in expression (1) hold simultaneously with probability greater than $1-\not$.

Expression (2) is derived from the results of Roy and Bose(1953) and is presented in Miller (1966). Expression (1) will generally give smaller confidence intervals. For $p=1$, expression ( $I$ ) is equal to expression (2) since

$$
\left(t_{n-1}^{\alpha / 2}\right)^{2}=F_{i, n-1}^{\alpha}
$$

For $\mathrm{p}$ 2,

$$
\left(t_{n-1}^{a / 2 p}\right)^{2}<F_{p, n-p}^{a}
$$

for small $n$. However, even when the $F$ value is less than $t$ value squared, the fact that expression (2) requires multiplication of the $F$ value by $p\left(\frac{n-1}{n-p}\right)$, results generally in the Bonferroni intervals being smaller. If in doubt one should compare $t_{n-1}^{\alpha / 2 p}$ to $\left(p F_{p, n-p}^{\alpha}\left(\frac{n-1}{n-p}\right)\right)^{1 / 2}$ to determine whether (1) or (2) will give the smaller intervals.

An example of case I taken from an actual experiment involves observations of the electrocardiogram. The ECG was recorded on magnetic tape and transformed by analogue to digital converter to numerical data. The mean and variance of the thirty-two points making up the 
p-wave are shown in Table I. Figure 1 shows a drawing of the average p-wave with confidence band derived using the formula for the Bonferroni intervals.

GASE II: COVARIANCE MATRIX KNOWN EXCEPT FOR A SCAIAR

The second case is a special case which assumes that the covariance matrix is known except for a scalar, that is,

$$
\Sigma=\sigma^{2} \Sigma^{\prime}
$$

where $\Sigma^{\prime}$ is known and $\sigma^{2}$ is unknown. For this case, Miller shows that the following expression gives Bonferoni intervals around each point such that the simultaneous probability that each point lies within the confidence band is greater than $1-\alpha$ :

$$
\text { (3) } \quad \mu_{i} \in \bar{y}_{i} \pm t_{p(n-1)}^{\alpha / 2 p} \frac{s}{\sqrt{n}} \sqrt{\sigma_{i}} \quad \text { i }=1, \ldots, p
$$

where $s^{2}=\frac{1}{\mathrm{p}-1} \sum_{j=1}^{n}\left(y_{j}-\overline{\mathrm{Y}}\right)^{\prime}\left(\Sigma^{\prime}\right)^{-1}\left(\mathrm{y}_{j}-\overline{\mathrm{Y}}\right)^{\prime}$

and $\sigma_{i i}$ is the ith diagonal element of the known matrix $\Sigma^{\prime}$. Alternatively, one could use:

(4) $\quad \mu_{i} \in \bar{y}_{i} \pm\left(\mathrm{pF}_{\mathrm{p}, p(n-i)}^{\alpha}\right)^{1 / 2} \frac{s}{\sqrt{n}} \sqrt{\sigma_{u}} \quad i=1, \ldots, \mathrm{p}$.

However, as before, the Bonferroni intervals will usually be smaller. Examples of this case are much less common. One example vould be where the curve was derived from observations on independent groups at each level of the incependent variable. In this case the covariance matrix would be diagonal: 
TABIE I

MEAN, VARTANCE, AND CONFIDENCE INTERVAL FOR AVERAGE P-WLVE

\begin{tabular}{|c|c|c|c|}
\hline point & mean & variance & Bonferroni intervals ${ }^{*}$ \\
\hline 1 & 168.92 & 3.34 & $168.92+.87$ \\
\hline 2 & 169.54 & 3.03 & $169.54+.83$ \\
\hline 3 & 169.58 & 5.02 & $169.58+1.06$ \\
\hline 4 & 170.02 & 5.24 & $170.02+1.08$ \\
\hline 5 & 171.48 & 4.70 & $171.48+1.03$ \\
\hline 6 & 173.62 & 6.73 & $173.62+1.23$ \\
\hline 7 & 176.60 & 6.82 & $176.60+1,24$ \\
\hline 8 & 179.30 & 6.99 & $179.30+1.26$ \\
\hline 9 & 180.72 & 4.98 & $180.72+1.06$ \\
\hline 10 & 181.34 & 4.80 & $181.34+1.04$ \\
\hline 11 & 180.40 & 7.84 & $180.40+1.33$ \\
\hline$\overline{12}$ & 179.46 & 5.40 & $179.46+1.10$ \\
\hline 13 & 180.52 & 10.91 & $180.52+1.57$ \\
\hline$\tilde{U}_{4}$ & 148.72 & 28.49 & $184.72+2.54$ \\
\hline 15 & 190.92 & 23.63 & $190.92+2.31$ \\
\hline 16 & 195.22 & 9.97 & $195.22+1.50$ \\
\hline 17 & 196.46 & 6.99 & $196.46+1.26$ \\
\hline 18 & 194.40 & 10.24 & $194.40+1.52$ \\
\hline 19 & 189.54 & 34.42 & $189.54+2.79$ \\
\hline 20 & 182.94 & 51.57 & $182.94+3.41$ \\
\hline 21 & 176.32 & 47.65 & $176.32+3.28$ \\
\hline 22 & 170.32 & 33.80 & $170.32+2.76$ \\
\hline 23 & 165.14 & 26.69 & $165.14+2.45$ \\
\hline 24 & 160.32 & 21.40 & $160.32+2.20$ \\
\hline 25 & 157.18 & 10.32 & $157.18+1.53$ \\
\hline 26 & 155.08 & 5.50 & $155.08+1.11$ \\
\hline 27 & 154.32 & 3.81 & $154.32+.93$ \\
\hline 28 & 154.22 & 3.24 & $154.22+.88$ \\
\hline 29 & 154.96 & 3.75 & $154.96+.92$ \\
\hline 30 & 155.56 & 3.64 & $155.56+.91$ \\
\hline 31 & 155.90 & 2.62 & $155.90+.77$ \\
\hline 32 & 156.42 & 2.66 & $156.42+.77$ \\
\hline
\end{tabular}




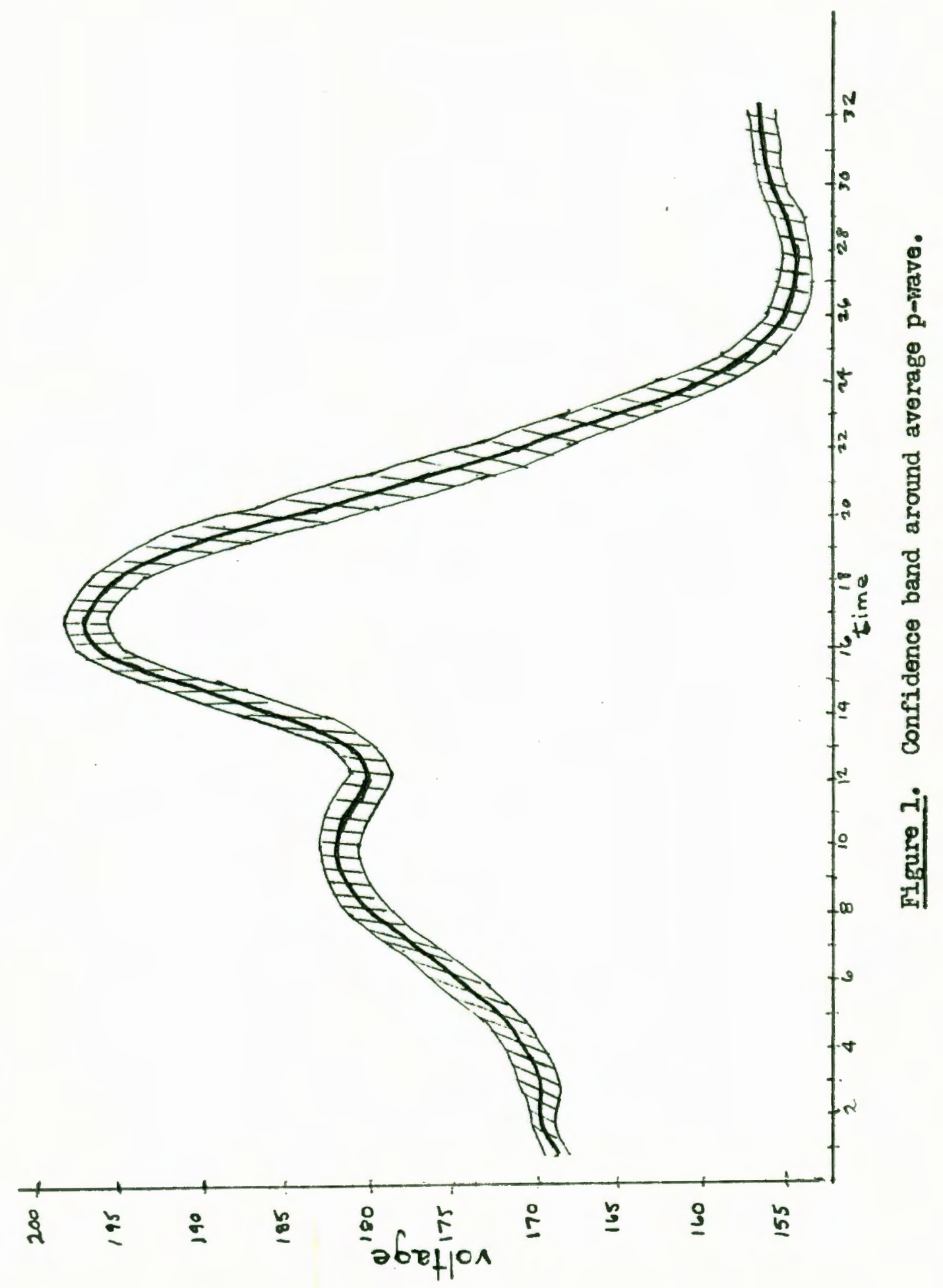




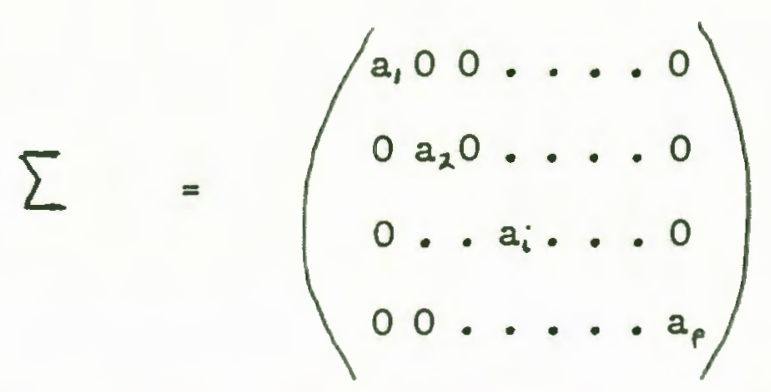

Assuming that the $a_{i}$ were equal or that they were all multiples of some common value $\left(a_{i}=c_{i} \sigma^{2}\right.$ where the $c_{i}$ are known), then the assumptions of case II would be met:

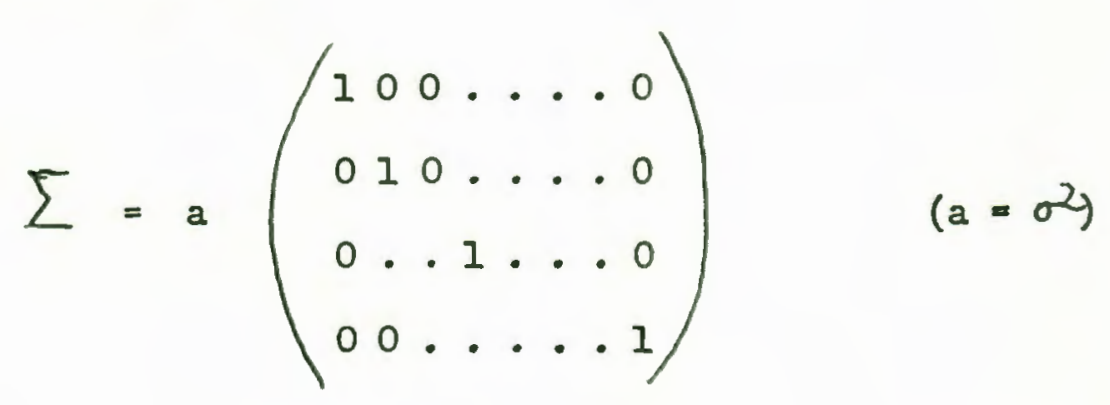

or

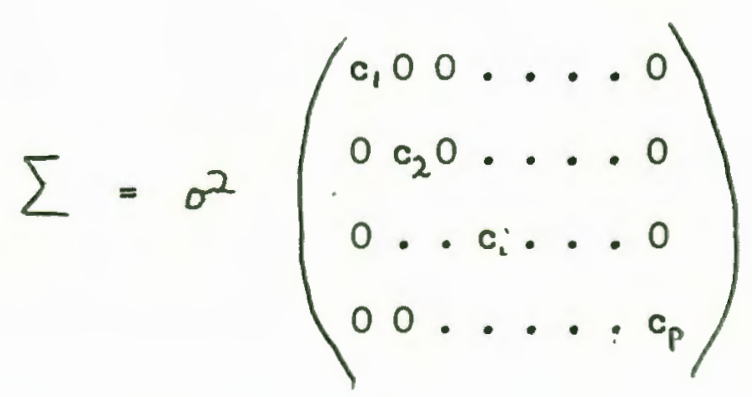

For instance, suppose that the $y$ represented performance measures on a memory task at four equally spaced dosages $(0.0 \mathrm{mg} / \mathrm{kg}$, $0.5 \mathrm{mg} / \mathrm{kg}, 1.0 \mathrm{mg} / \mathrm{kg}, 1.5 \mathrm{mg} / \mathrm{kg}$ ) of a drug which is a synthetic analogue of $\Delta^{9}$-tetrahydrocamnabinol (syn. $\Delta^{9}-\mathrm{THC}$ ). If the experimenter had considerable experience with data derived from these dosage levels of this drug, he could possibly arrive at estimates of the $a_{i}$. Suppose that he noted twice as much variance in the perform- 
ance at the upper two levels of the drug as at the lower two. Then,

$$
\Sigma=\sigma^{2}\left(\begin{array}{llll}
1 & 0 & 0 & 0 \\
0 & 1 & 0 & 0 \\
0 & 0 & 2 & 0 \\
0 & 0 & 0 & 2
\end{array}\right)
$$

assuming that independent groups were used at each dosage level.

Table II shows the raw scores for each of ten subjects used at each dosage level (data simulated). Table III gives the values of $\bar{y}_{i}$ and the confidence interval around each point which was computed using expression (3) ( $s^{2}$ was calculated to be 56.43 and $\alpha$ was set at .05 ). Figure 2 shows the confidence band around the mean curve. 


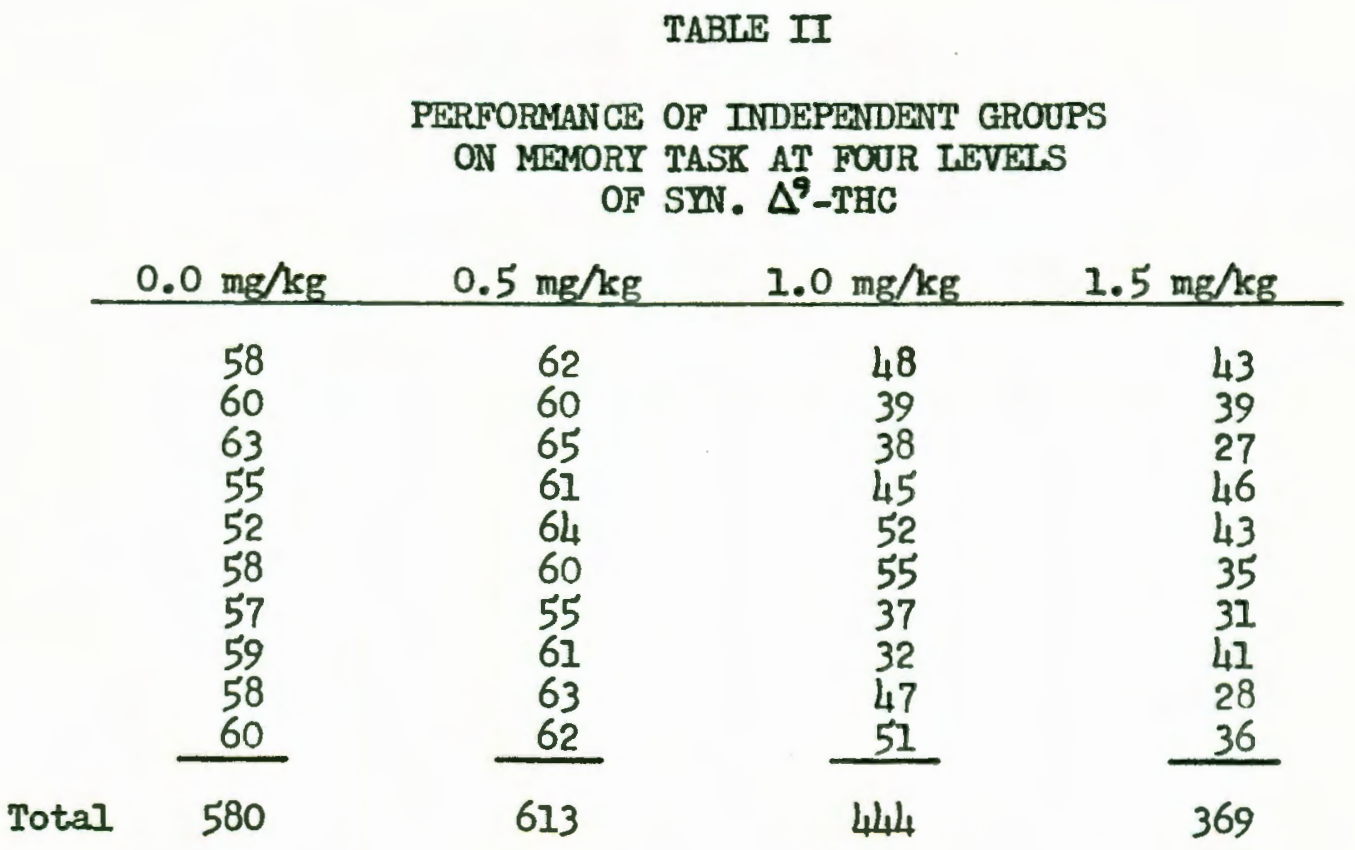

TABLE III

AVERAGE SCORE AND CONFIDENCE INTERVAL AROUND EACH POINT FOR

STN. $\Delta^{9}-$ THC

\begin{tabular}{|c|c|c|}
\hline point & $\overline{y_{i}}$ & Bonferroni confidence \\
\hline 1 & 58.0 & $58.0+6.23$ \\
\hline 2 & 61.3 & $61.3+6.23$ \\
\hline 3 & 44.4 & $44.4+8.85$ \\
\hline 4 & 36.9 & $36.9+8.85$ \\
\hline
\end{tabular}




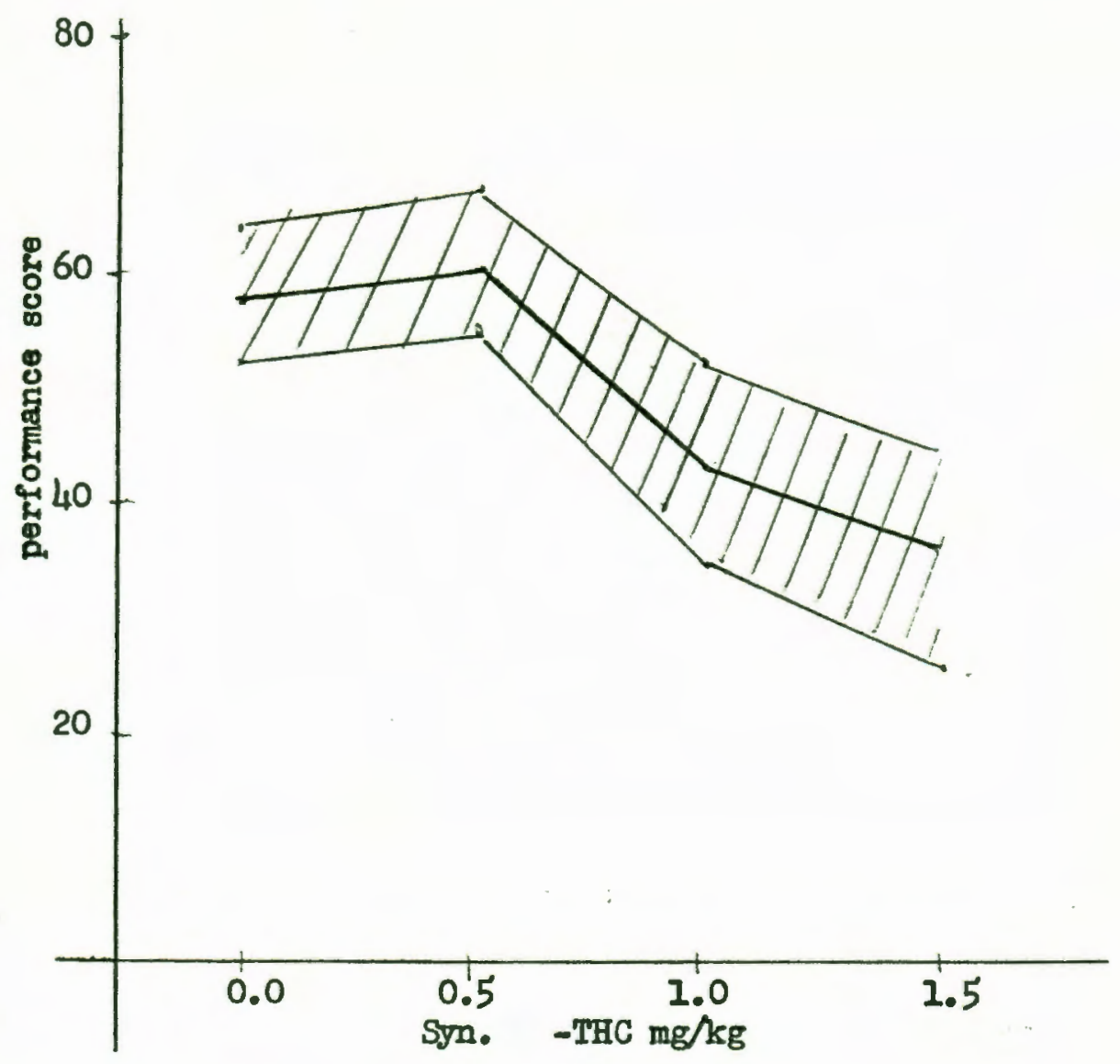

Figure 2. Confidence band around performance scores at four dosage levels of a synthetic analoque of $\triangle$-THC. 
III. FINDTNG A CONFTDENCE BAND FOR THE DIFFERENCE BETWEEN TWO CURVES

The second type of analysis to be considered is that of finding a confidence interval around the difference between two or more curves. This analysis has as its univariate analogue the comparison of the means of two groups, or finding a confidence interval for the difference between two groups. In the univariate case, one finds the mean for each of the two samples and then finds the confidence interval for the difference using the folloving formula:

$$
\begin{gathered}
\mu_{d} \in\left(\bar{y}_{1}-\bar{y}_{2}\right) \pm t_{n_{1}+n_{2}-2}^{a / 2} \bar{\sigma}_{d} \\
\text { where } \bar{\sigma}_{d}=\sqrt{\frac{\left(n_{r}-1\right) s^{2}+\left(n_{2}-1\right) s^{2}}{n_{1}+n_{2}-2}} \sqrt{\frac{1}{n_{1}}+\frac{1}{n_{2}}}
\end{gathered}
$$

In the case of curve comparisons, one wishes to find a confidence band on the difference between two average curves. An example would be comparing the average ECG of an animal before administration of a drug to the average ECG after administration (see below).

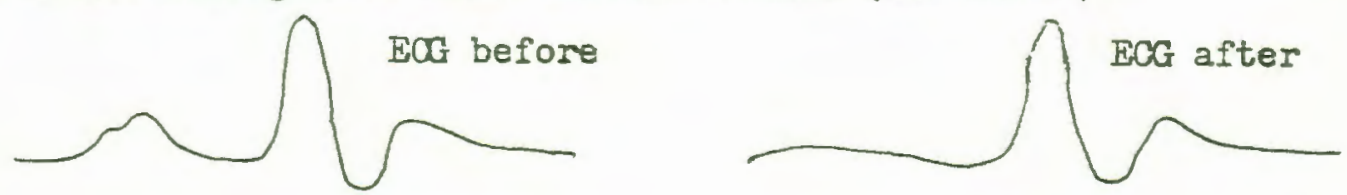
Gurrent methods of making such comparisons are visual inspection of the graphs, analysis of variance or Hotelling's $\mathrm{T}^{2}$.

Visual inspection has been commonly used in the comparison of waveforms like the ECG. In such waveforms there are often regularly occurring phenomena which can oasily be identified by visual inspection as in the above drawings. The difficulty with visual inspection for curve comparison is similar to inspection of single values in the uni- 
variate case: the variance about each point may be so large, that apparent differences are not statistically significant; that is, the differences could be due to chance alone.

Existing statistical techniques, on the other hand, mas obfuscate real differences, hiding differences that would be significant by the alternative techniques to be presented. Use of the analysis of variance for the comparison of two curves may often hide real differences. Analysis of variance can be used for the comparison of curves by considering each point as one colum in the analysis, and each sample of curves as a row. The design would be interpreted as a two-way ANOVA, with repeated measures on the row variables. Only an overall difference will be apparent. If a difference exists in a small portion of the total number of points, it may be that there will be no significant difference between rows. Differences in different segments will show up as significant interaction, but will not indicate at which points the differences occur, thus obscuring a difference that would be obvious to visual inspection.

Another difficulty with the use of ANOVA presents itself when the points of the curve represent repeated measures on the same subjects. In using ANOVA, it is assumed that the variance-covariance matrix has comound symetry, that is, that:

$$
\Sigma=\sigma^{2}\left(\begin{array}{lllllll}
1 & \rho & \rho & \cdot & \cdot & \cdot & \rho \\
\rho & 1 & \rho & \cdot & \cdot & \cdot & \rho \\
\cdot & \cdot & \cdot & \cdot & \cdot & \cdot & \cdot \\
\rho & \rho & \cdot & \cdot & \cdot & \cdot & 1
\end{array}\right)
$$


In the case of independent groups, $\rho=0$, and this assumption amounts to saying that the treatment means have equal variance. Box (1954) has shown that in the usual ANOVA (not repeated measures), the F-test is relatively insensitive to violations of the assumption of compound synmetry. However, in the case of repeated measures (or whenever observations are correlated) violation of this assumption will result in a positive bias (an $F$ value which is too low).

Therefore, before performing ANOVA with repeated measures, it is necessary to test that $\Sigma$ has the above form. This involves testing the hypothesis that the covariance matrices for each sarple are equal and then testing that the pooled matrix has variances equal to the average variance of the pooled estimate and covariances equal to the average covariance of the pooled estimate (Winer, 1971). The computations involve finding the matrix of the average variance and covariances, the determinant of the pooled estimate, and the natural log of the determinant. If the matrix is found to have the required form, then one can proceed with the ANOVA. This procedure of determining if $\Sigma$ has the proper form is already more difficult computationally than proceeding with Miller's simultaneous techniques to be presented below. Also, if one can proceed with ANOVA, this will still involve the limitations described above regarding the analysis of differences in different portions of the curves.

If the matrix does not have the required shape, the usual procedure is to use Hotelling's $T^{2}$ to determine if there are coluran differences (winer, 1971). However, this is a test of colum effects only, not of row differences or interaction. When considering differences between two or more curves like the ECG or spectral sensitivity 
curves, one already knows that there are differences in level between the points of the curve inherent in the shape of the curre. One would be interested in differences in shape between different groups, that is, in row differences and interaction.

The $T^{2}$ test can still be used to test for differences between curves by using the procedure described in Morrison's Multivariate Statistical Methods (1967). Computation of $\mathrm{T}^{2}$ is difficult, involving the calculation of a matrix inverse. The confidence regions that can be determined are p-dimensional ellipsoids. With $\mathrm{p}>3$, these regions become difficult to visualize. Also, this test, like ANOVA, gives just an overall difference rather than a difference at each point.

An alternative technique presented by Miller provides for the determination of a confidence interval for the difference between the means of each co-ordinate of the curve, allowing one to distinguish at which points the curves differ and at which they are the same. Roy and Bose (1953) developed this method by detemining the point by point implication of the p-dimensional ellipsoid derived from Hotelling's $\mathrm{T}^{2}$. The computations involved in this technique are much simpler than those required for Hotelling's $T^{2}$ ( $T^{2}$ does not have to be calculated) or those required for ANOVA. All that is required is an estimate of the variance about each point for case I or an estimate of the unknown scalar for case II. Since case I makes no assumption about the shape of the covariance matrix, there is no need to make the tests for compound symmetry. 
CASE I: COVARIANCE MATRIX UNKNOWN

In the general case where the covariance matrix is unknown, the formula for finding the confidence interval about the difference between two curves based on the Bonferoni inequality is:

$$
\mu_{1 i}-u_{2 i} \in \bar{y}_{1 i}-\bar{y}_{2 i}=t_{2(n-1)}^{\alpha / 2 p}\left(\frac{2 s_{i}^{2}}{n}\right)^{1 / 2}
$$

where $\mathrm{s}_{i}^{2}$ is the pooled estimate of the variance at each point and where the $\bar{Y}_{1 i}$ are co-ordinate means of the first sample and the $\bar{y}_{2 i}$ are the co-ordinate means of the second sample.

An example of this case would be the comparison of the p-wave before administration of a drug to the p-wave after administration. Data from an actual experiment gave the values shown in Table IV for the mean and variance of the p-waves before and after administration of $\triangle$-THC. A sample of fifty was taken in each case. The difference between the two curves and the confidence band about the difference is also shown in table IV. Figure 3 shows the average curves of the two curves being compared. Shown in figure 4 is the confidence band about the difference between the two curves. The two curves are different at all points except 24 and 25 where the confidence bands include zero, with the largest differences occurring between points $\mathrm{ll}_{4}$ and 20. This analysis provides statistical validation of what would be obvious to visual inspection.

\section{CASE II: COVARIANCE MATRIX IKWWN EXCEPT FOR A SCALAR}

The second case assumes that the covariance matrix is known except for a constant ( $\Sigma=\sigma^{2} \Sigma^{\prime}$ where $\Sigma^{\prime}$ is known and $\sigma^{2}$ uniknown). 
TABLE IV

CONEIDENCE BAND ABOUT DIFFERENCE BETWEEN AVERAGE P-WAVES BEFORE AND AFTER

ADMINISTRATION OF -THC

\begin{tabular}{|c|c|c|c|c|c|c|c|}
\hline pt. & $\bar{y}_{1 i}$ & $\bar{y}_{2 i}$ & $\bar{y}_{1 i}-\bar{y}_{2 i}$ & $s_{1 i}^{2}$ & $s_{2 i}^{2}$ & pooled $s_{i}^{2}$ & $\begin{array}{l}\text { confidence } \\
\text { interval * }\end{array}$ \\
\hline & & & & & & & \\
\hline $\begin{array}{l}1 \\
2\end{array}$ & $\begin{array}{l}168.92 \\
169.54\end{array}$ & $\begin{array}{l}159.54 \\
159.94\end{array}$ & $\begin{array}{l}9.48 \\
9.60\end{array}$ & 3.34 & $\begin{array}{r}8.58 \\
13.98\end{array}$ & $\begin{array}{l}5.96 \\
8.50\end{array}$ & $\begin{array}{l}9.48+1.59 \\
9.60+1.90\end{array}$ \\
\hline 3 & 169.58 & 159.58 & 10.00 & 5.02 & 10.29 & 7.66 & $10.00+1.80$ \\
\hline 4 & 170.02 & 159.42 & 10.60 & 5.24 & 6.53 & 5.89 & $10.60+1.58$ \\
\hline 5 & 17.48 & 159.68 & 11.80 & 4.70 & 5.20 & 4.95 & $11.80+1.45$ \\
\hline 6 & 173.62 & 159.78 & 13.84 & 6.73 & 6.54 & 6.64 & $13.84+1.68$ \\
\hline 7 & 176.60 & 158.98 & 17.62 & 6.82 & 7.98 & 7.40 & $17.62+1.77$ \\
\hline 8 & 179.30 & 157.96 & 21.34 & 6.99 & 9.39 & 8.19 & $21.34+1.87$ \\
\hline 9 & 180.72 & 156.82 & 23.90 & 4.98 & 18.88 & 11.93 & $23.90+2.25$ \\
\hline 10 & 181.34 & 155.66 & 25.68 & 4.80 & 22.72 & 17.84 & $25.68+2.24$ \\
\hline 17 & 180.40 & 154.46 & 25.94 & 7.84 & 28.87 & 18.35 & $25.94+2.79$ \\
\hline 12 & 179.46 & 152.52 & 26.94 & 5.40 & 42.05 & 23.72 & $26.94+3.17$ \\
\hline 13 & 180.52 & 149.46 & 31.06 & 10.91 & 51.27 & 31.09 & $31.06+3.63$ \\
\hline II & 184.72 & 246.70 & 38.02 & 28.49 & 85.89 & 57.19 & $38.02+4.93$ \\
\hline 15 . & 190.92 & 143.88 & 47.04 & 23.63 & 93.70 & 58.66 & $47.04+4.99$ \\
\hline 16 & 195.22 & य2.14 & 53.08 & 9.97 & 93.80 & 51.89 & $53.08+4.70$ \\
\hline 17 & 196.46 & 14.36 & 55.10 & 6.99 & 61.95 & 34.47 & $55.10+3.82$ \\
\hline 18 & 194.40 & 142.16 & 52.24 & 10.24 & 43.44 & 26.84 & $52.24+3.37$ \\
\hline 19 & 189.54 & $\mu_{43} 30$ & 46.24 & 34.42 & 50.91 & 42.67 & $46.24+4.26$ \\
\hline 20 & 182.94 & 145.24 & 37.70 & 51.57 & 53.86 & 52.71 & $37.70+4.73$ \\
\hline 21 & 176.32 & 148.16 & 28.16 & 47.65 & 50.14 & 48.89 & $28.16+4.56$ \\
\hline 22 & 170.32 & 150.46 & 19.86 & & 43.40 & 38.60 & $19.86+4.05$ \\
\hline 23 & 165.14 & 153.64 & 11.50 & 26.69 & 38.32 & 32.51 & $21.50+3.72$ \\
\hline 24 & 160.32 & 157.10 & 3.22 & 21.40 & 23 & 32.31 & $3.22+3.71$ \\
\hline 25 & 157.18 & 160.34 & -3.15 & 10.32 & 48.15 & 29.23 & $-3.15+3.53$ \\
\hline 26 & 155.08 & 162.66 & -7.58 & 5.50 & 63.54 & 34.52 & $-7.58+3.83$ \\
\hline 27 & 154.32 & 164.64 & -10.32 & 3.81 & 58.97 & 31.39 & $-10.32+3.65$ \\
\hline 28 & 154.22 & 166.88 & -12.66 & 3.24 & 47.74 & 25.49 & $-12.66+3.29$ \\
\hline 29 & 154.96 & 168.84 & -13.88 & 3.75 & 28.72 & 16.24 & $-13.88+2.63$ \\
\hline 30 & 155.56 & 169.80 & -14.24 & 3.64 & 22.86 & 13.25 & $-14.24+2.37$ \\
\hline 31 & 155.90 & 170.22 & -14.32 & 2.62 & 20.79 & 11.7 & $-14.32+2.23$ \\
\hline 32 & 156.42 & 170.06 & -13.64 & 2.66 & 26.87 & 14.76 & $-13.64+2.50$ \\
\hline & & & & & & & $\alpha=.0$ \\
\hline
\end{tabular}


20

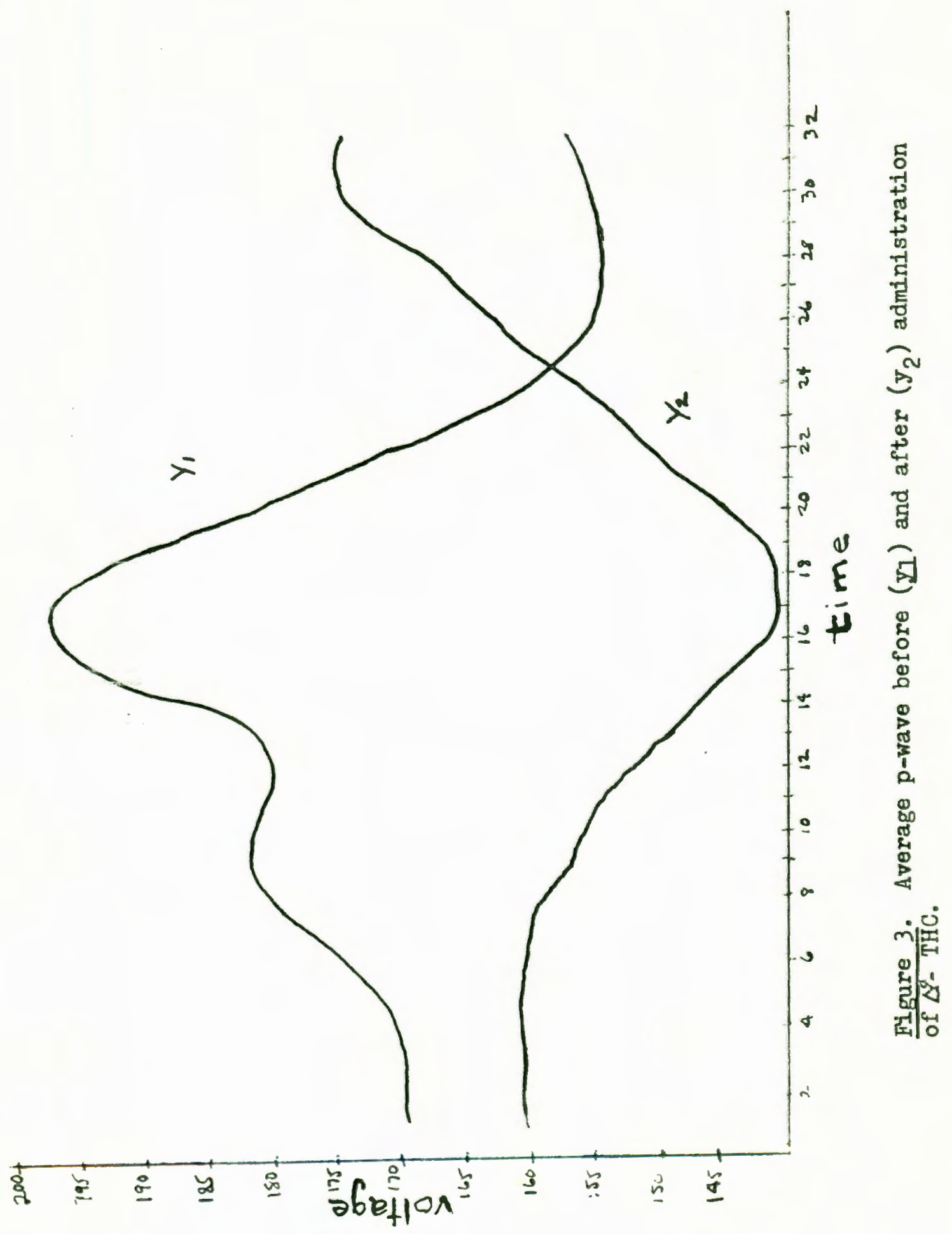




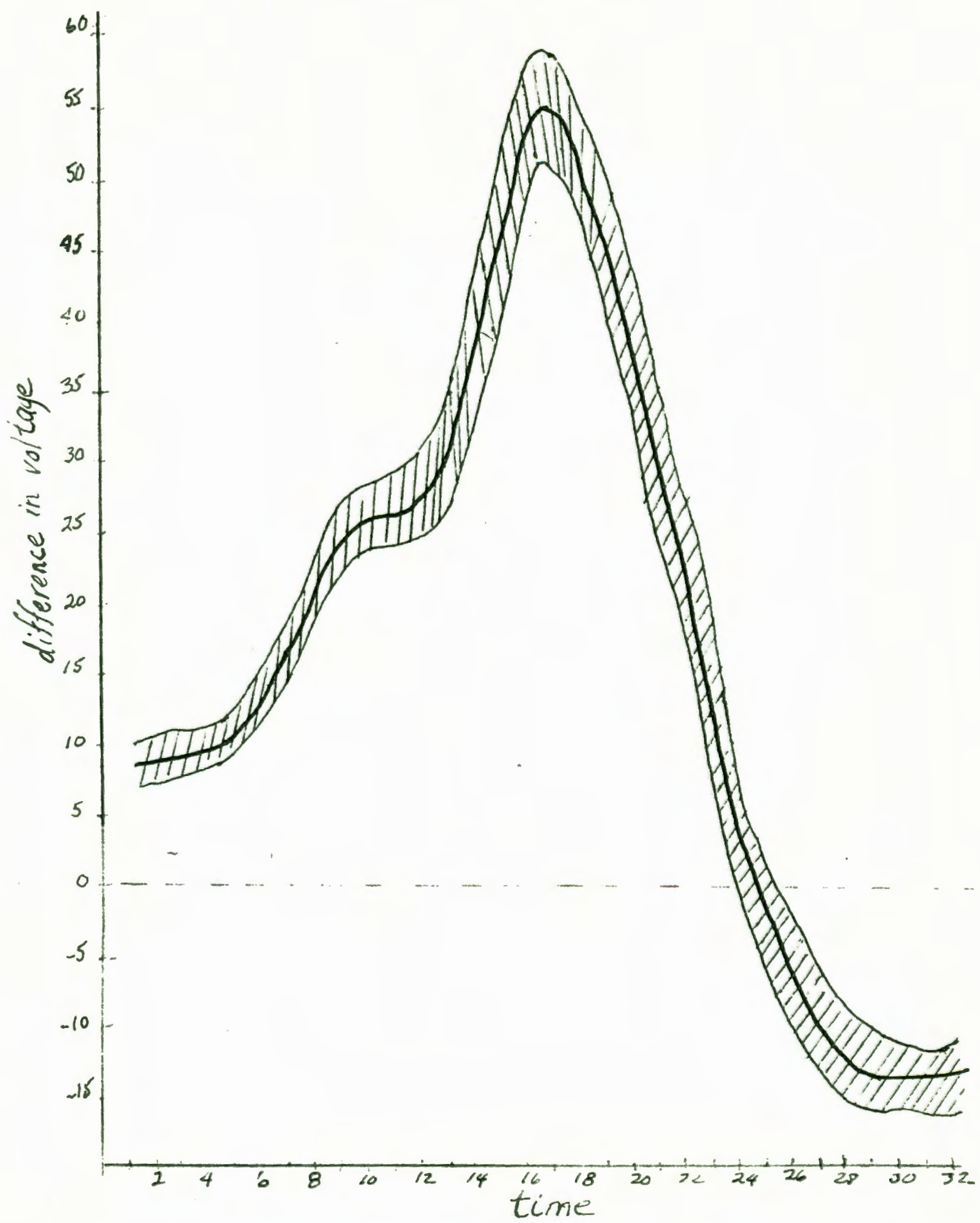

Figure 4. Confidence band about difference between average p-waves shown in figure 3 . 
For this case the confidence bands for the difference between two curves can be found by the use of the following:

$$
\mu_{1 i}-\mu_{2 i} \in \bar{y}_{1 i}-\bar{y}_{2 i} \pm t_{2 p(n-1)}^{\alpha / 2 p}\left(\frac{2 s^{2} \sigma_{i i}}{n}\right)^{1 / 2}
$$

where $s^{2}=\frac{1}{2 \mathrm{p}(n-1)}\left(\sum_{i=1}^{n}\left(\underline{y}_{i}-\bar{y}_{1}\right)^{\prime}\left(\Sigma^{\prime}\right)^{-1} \frac{\left(y_{i} i\right.}{n}-\bar{y}_{1}\right)$

$$
\left.+\sum_{j=1}^{n}\left(\bar{y}_{2 i}-\underline{y}_{2}\right)^{\prime}\left(\Sigma^{\prime}\right)^{-1}\left(\bar{y}_{2 i}-\bar{y}_{2}\right)\right)
$$

and where $\sigma_{i i}$ is the ith diagonal element of the known matrix $\Sigma^{\prime}$.

An example of this case would be the comparison of the two doseresponse curves shom in figure 4 where syn. $\Delta^{9}$-THC was the drug used in obtaining curve 1 and $\Delta^{\mathcal{G}}$-THC was used in obtaining curve 2. Assuming that independent groups were used at each dosage level and that the variance was known to be twice as large at the two higher levels as at the two lower levels for both drugs, the covariance matrix would have the form:

$$
\Sigma=\sigma^{2}\left(\begin{array}{llll}
1 & 0 & 0 & 0 \\
0 & 1 & 0 & 0 \\
0 & 0 & 2 & 0 \\
0 & 0 & 0 & 2
\end{array}\right)
$$

The raw data for the scores obtained using the first drug is shown in table II, while table $\nabla$ gives the raw scores for drug 2 (data simulated in both cases). Table VI gives the mean difference between the curves at each dosage level and a confidence band about this difference. Figure 5a shows the two average curves and figure $5 b$ the confidence band for the difference between the two curves. The two curves differ significantly at dosage levels 1.0 and $1.5 \mathrm{mg} / \mathrm{kg}$. There was no significant difference at 0.0 and $0.5 \mathrm{mg} / \mathrm{kg}$. 


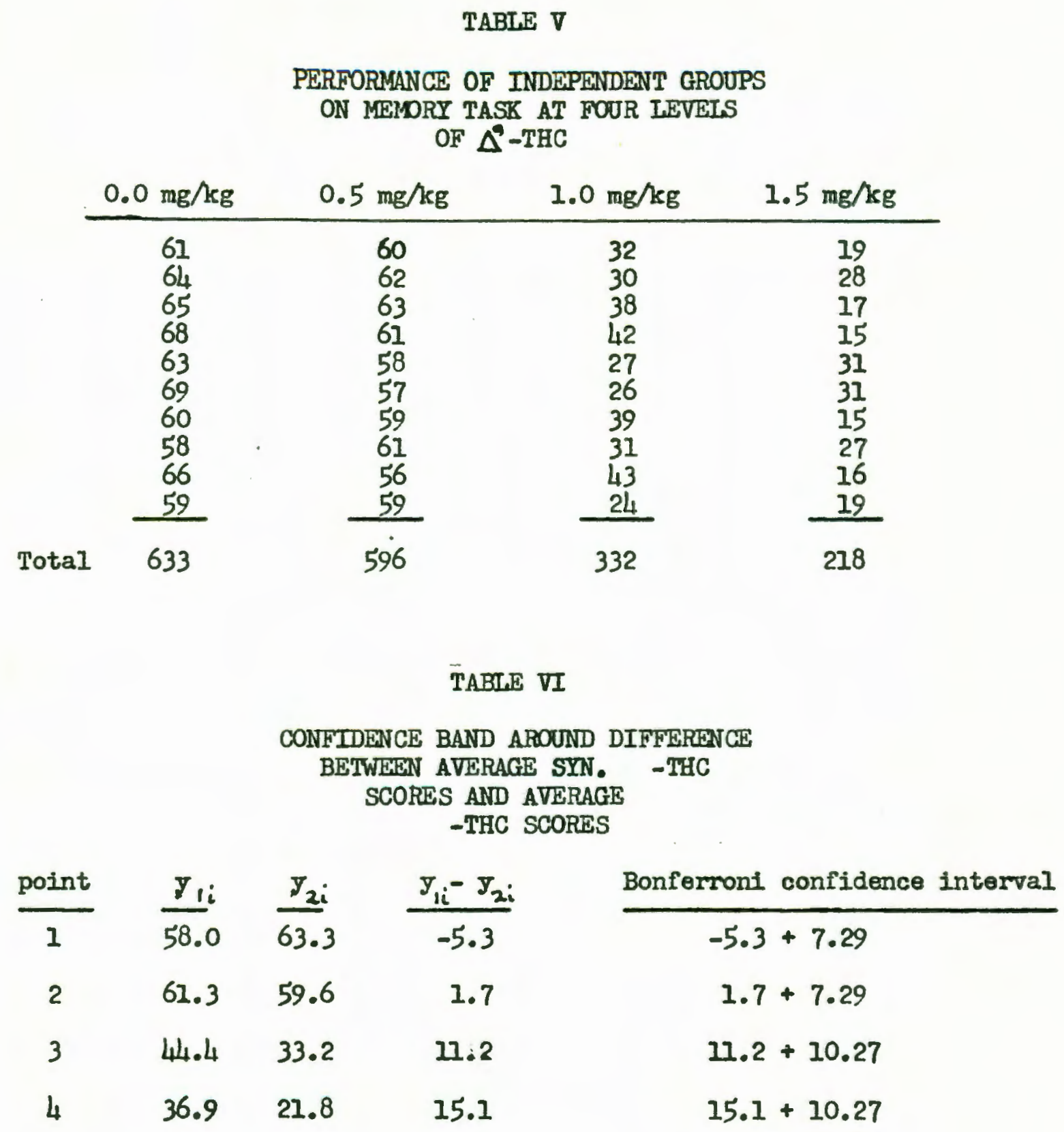




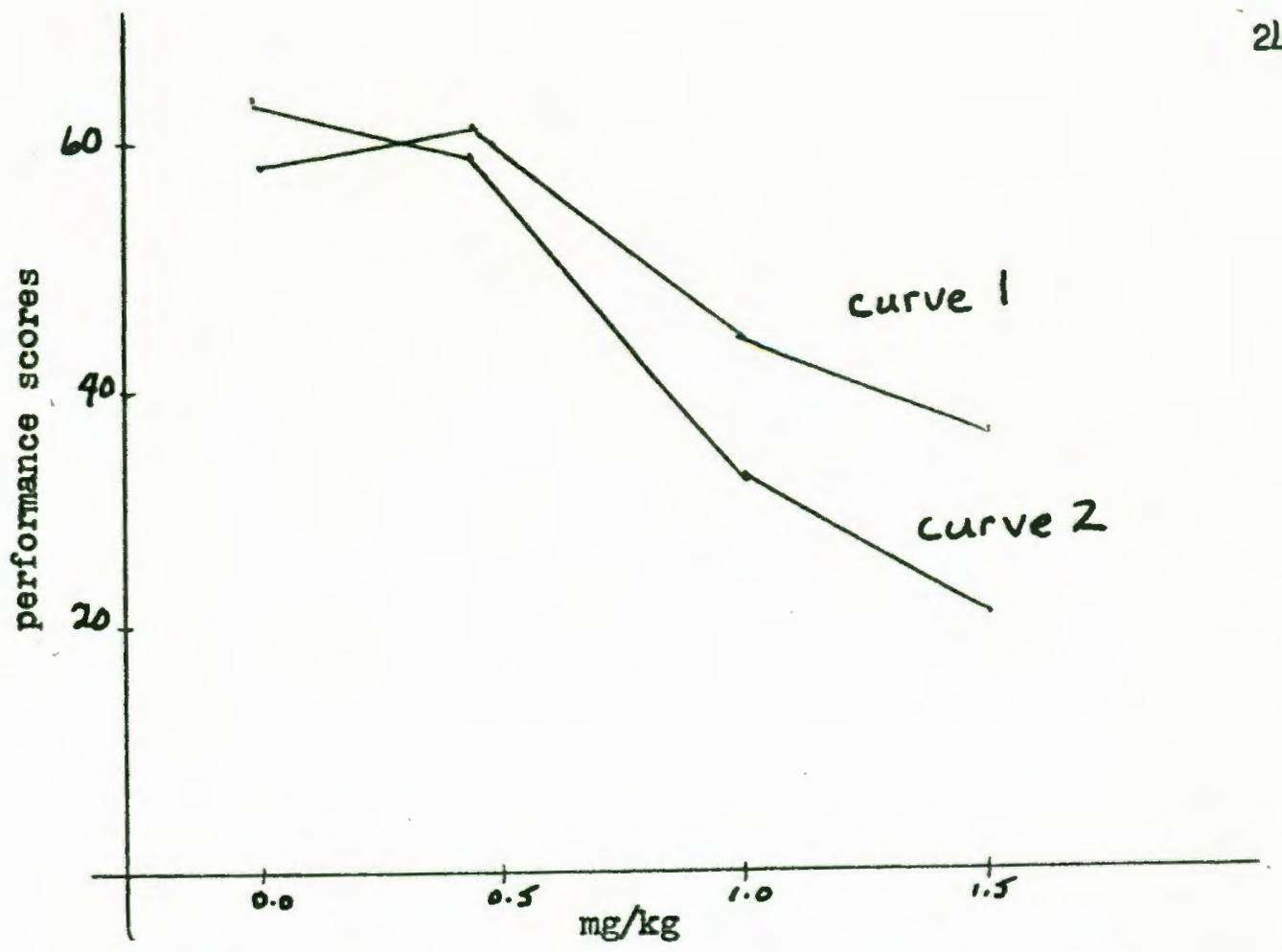

Figure 5a. Curve $I$ shows average performance score at four dosage levels of syn. $\Delta^{9}$-THC. Curve 2 shows the same for $\Delta-$ THC.

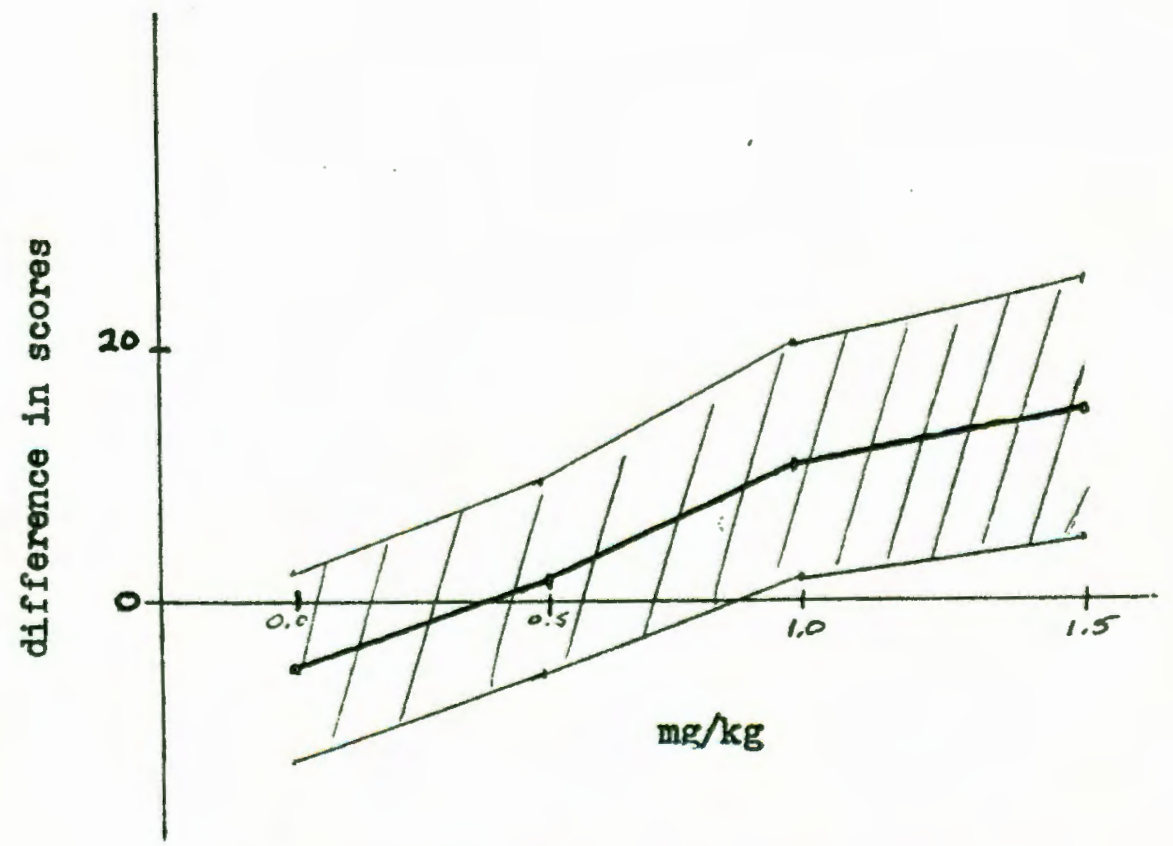

Figure 5b. Confidence band about the difference betwen the two curves shown in figure 5 . 


\section{DECOMPOSITION OF A CURVE INTO ITS COMPONENTS}

This section deals with a type of analysis which attempts to define the shape of a curve or the form of relationship between the independent variable and the dependent variable. First to be discussed will be the general idea of a component of a curve as being a Iinear combination of co-ordinate means. Next to be presented will be the determination of orthogonal components and two special sets of orthogonal components known as orthogonal polynomials and Haar functions. Finally, techniques for making simultaneous tests on the significance of a set of components will be outlined.

Besides finding a confidence band about a mean curve or mean difference between curves, one may wish to analyze the shape of a curve. This type of analysis has no univariate analogue since one cannot talk about shape or form in one dimension. In the case of curves, one would like to be able to say, for instance, that curve $6 a$ (figure 6) shows a difference in level between first half and second half, while curve $6 \mathrm{~b}$ shows a linear trend. The means of making this type of analysis depend on making comparisons between weighted combinations of the co-ordinate means. For example, to compare the first half of curve $6 a$ to the second half one could look at the difference between the sum of means of the first half and the sum of means of the second half:

$$
\left(I \cdot y_{1}+I \cdot y_{2}\right)-\left(I \cdot y_{3}+I \cdot y_{4}\right)
$$



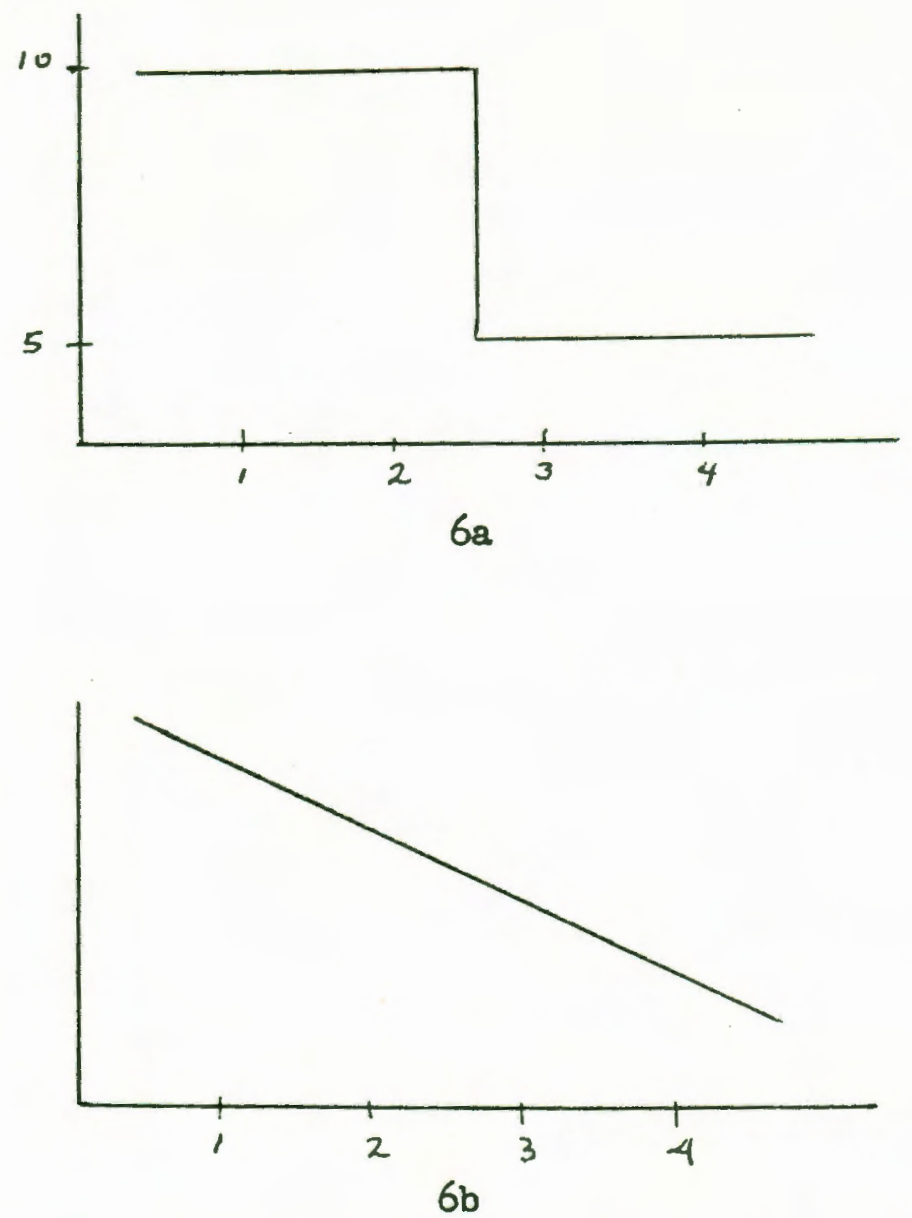

Figure 6. Curve $6 a$ shows a difference in level between the first half and the second half. Curve $6 \mathrm{~b}$ shows a linear trend. 
Then this difference can be tested for statistical significance. Thinking of the co-ordinate means as being colum means in an analysis of variance, the statistical test for the significance of combinations of co-ordinate means has close relationship to the test of colum effects in one-way ANOVA.

A component of the sum of squares for comordinate means is defined by

$$
S S_{c}=\frac{\left(c_{1} y_{1}+c_{2} y_{2}+c_{3} y_{3}+\ldots . . . . .+c_{p} y_{p}\right)^{2}}{n\left(c_{1}^{2}+c_{2}^{2}+c_{3}^{2}+\ldots . . .+c_{p}^{2}\right)} \quad \text { where } \sum_{i=1}^{p} c_{i}=0
$$

or, in vector notation where $\underline{c}^{1}=\left(c_{1}, c_{2}, c_{3}, \ldots . ., c_{p}\right)$

$$
S s_{c}=\left(\underline{c}^{\prime} \underline{y}\right)^{2} / \underline{n}^{\prime} \underline{c} .
$$

In the foregoing exarmle, $c_{1}=c_{2}=1, c_{3}=q_{4}=-1$, and $s s_{c}=25 / n$. A component of the sum of squares has one degree of freedom because it represents the squared difference between two basic observations each of which is a weighted sum of treatment means (winer, 1971). The expected value of a component of sum of squares is:

$$
E\left(S S_{c}\right)=\sigma_{\epsilon}^{2}+n / 2\left(\underline{c}^{\prime} y\right)^{2}
$$

Under the hypothesis that $\underline{c}^{\prime} y=0$, that is, that the weighted difference equals zero,

$$
E\left(S S_{c}\right)=\sigma_{t}^{2}
$$

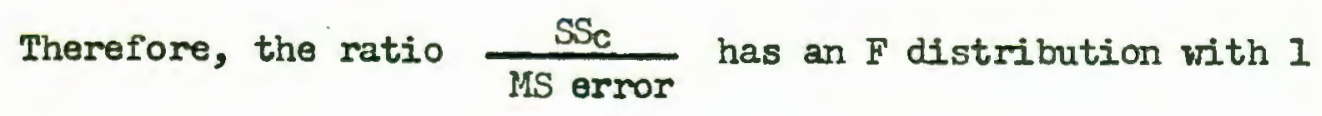
and $p(n-1)$ degrees of freedom.

Many different combinations of the co-ordinate means could be 
derived meeting the requirement that $\Sigma_{c_{1}}=0$. However, only certain sets of these combinations will provide non-overlapping information about the shape of the curve. Returning to curve $6 a$, one could compare the first co-ordinate mean to the fourth by taking the combination:

$$
1 \bar{y}_{1}+0 \bar{y}_{2}+0 \bar{y}_{3}-1 \bar{y}_{4}
$$

However, the information obtained from doing so would be rechundant with that obtained in taking the combination in (7). On the other hand, one could look at the difference between the first co-ordinate and the second by taking the combination:

$$
1 \bar{y}_{1}-1 \bar{y}_{2}+0 \bar{y}_{3}+0 \bar{y}_{4}
$$

This would not overlap with (7) since (7) tells us nothing about relationships in the first half, only about relationships between the first half and the second.

An easy means of determining whether two linear combinations are uncorrelated and therefore provide non-overlapping information is to take the vector product $\underline{c}_{1}^{\prime} c_{2}$, where $\underline{c}_{1}^{\prime}$ is the set of weights for one combination and $\mathrm{c}_{2}$ is the set of weights for the other. If $\underline{c}_{1}^{\prime} \underline{c}_{2}=0$, the combinations are orthogonal (i.e., uncorrelated). For example,

$$
(1,1,-1,-1) \quad(1,0,0,-1)=2 \text {, }
$$

and these tro combinations would not be orthogonal, while

$$
(1,1,-1,-1) \quad(1,-1,0,0)=0,
$$

and these combinations would be orthogonal.

If the combinations are orthogonal, then the sums of squares for 
the components will also be orthogonal. For a curve comprised of $p$ points ( or a design with $\mathrm{p}$ columns), one can determine p-l components all of which are orthogonal to each other. Each component of the sums of squares for these p-1 components covers a different portion of the total variation of the co-ordinate means. The sum of these p-l components of the sum of squares will account for 271 the variation between groups. Non-orthogonal components do not have this additive property. (Winer, 1971)

Therefore, by taking orthogonal linear combinations of the co-ordinate means one can arrive at k-1 non-overlapping bits of information about the shape of the curve. Two sets of components which provide useful information about the shape of a curve are orthogonal polynomials and Haar functions. The first to be discussed will be orthogonal polynomials.

\section{ORTHOGONAI POLTNOMIAIS}

The only method discussed in most texts on psychological statistics for the charaterization of the components of a curve is the method of orthogonal polymomials (eg. Hays, 1973). The weights to be used in taking linear combinations of means for finding orthogonal components have been tabled. The weights have been chosen so that each of the p-1 sets of coefficients in combination with the coordinate means represent exactly one polynomial trend in the data. The first set of coefficients allows one to test for a linear trend, the second for a quadratic trend, the third for a cubic trend, the fourth for a quartic trend and so on. (See figure 7.) 


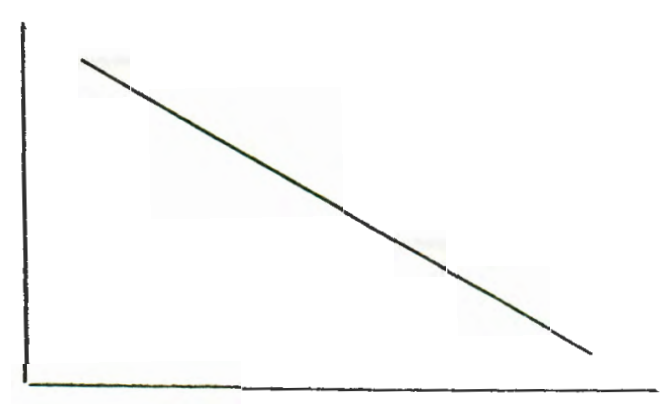

linear

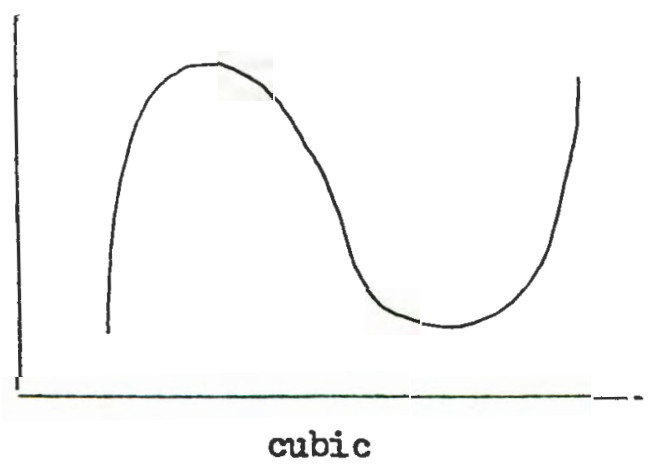

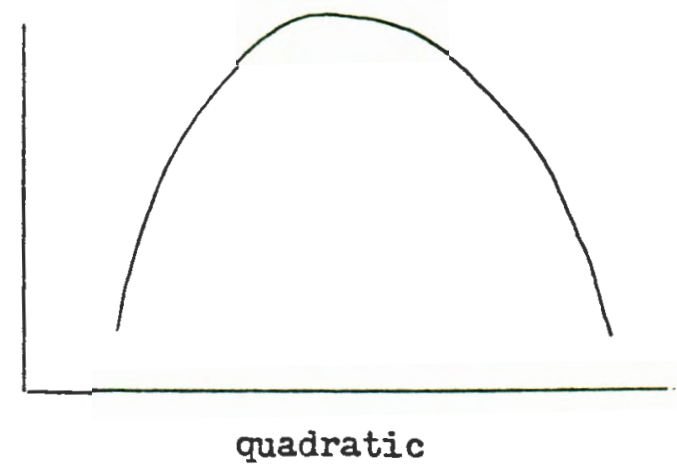

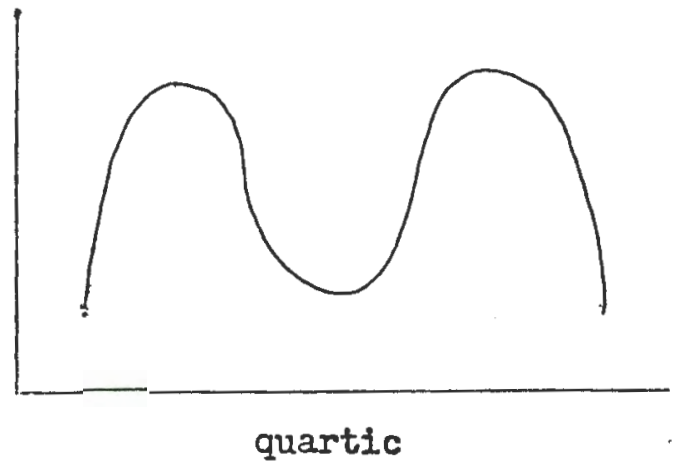

Figure 7. Curves show the first four polynomial trends. 
A curve may have one or more significant trends, up to $\mathrm{p}-1$, or it may have no significant trends. For example, a curve of shape 8a (see figure 8) would shon a small linear trend; a curve of shape $8 \mathrm{~b}$ would show a larger linear trend; curve $8 \mathrm{c}$ would show a quadratic trend and no other; and curve $8 \mathrm{~d}$ would show a significant linear trend as well as a significant quadratic trend.

Difficulty with this method arises if the curve involves a number of significant trends. This can occur even if the curve is relatively simple but non-symmetric as in the case of figure 9. Suppose that for this curve there were 10 observations at each point and that the mean squares for error (MS error) was equal to 40.0. The symbol $\psi_{p_{i}}$ will be defined as follows:

$$
\psi_{f_{i}}=\mathrm{p}_{i}^{\prime} \overline{\mathbf{z}}
$$

where $\underline{p}_{i}$ is the set of orthogonal polynomial coefficients for the ith component. The $S_{S^{\prime}}{ }^{\prime}$ for the $\psi_{P_{i}}$ were found to be:

$$
\begin{array}{ll}
\operatorname{SS}\left(\psi_{p_{i}}\right)=2881 & \operatorname{ss}\left(\psi_{P_{+}}\right)=0 \\
\operatorname{SS}\left(\psi_{P_{2}}\right)=0 & \operatorname{SS}\left(\psi_{P_{3}}\right)=468 \\
\operatorname{SS}\left(\psi_{P_{3}}\right)=969 &
\end{array}
$$

With an $\alpha$ of .05 and with $I$ and 72 degrees of freedom, the ratio of SS $\left(\psi_{p_{i}}\right) /$ MS error would be compared to the $F$ value of 3.98. Trends 1,3 , and 5 would be significant. It is also quite Iikely that the seventh trend would also be significant. However, coefflcients for trends beyond the fifth are not usually tabled.

The problem with this method lies in the physical meaning to accord to the various signtfican trends beyond quadratic. This 


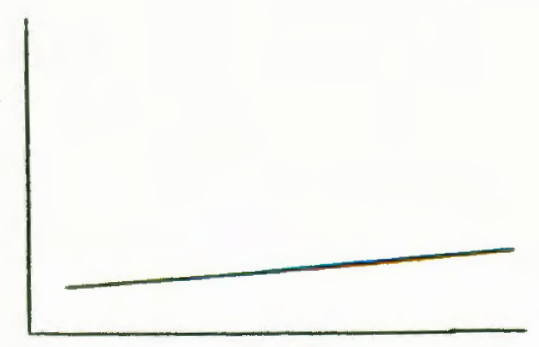

$8 a$

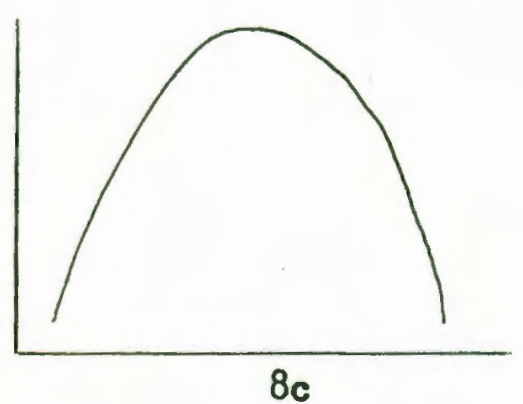

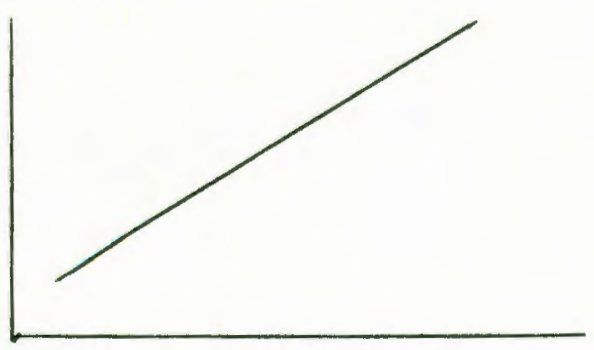

$8 b$

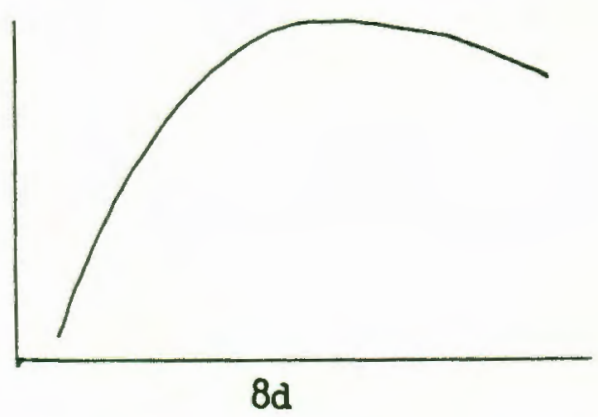

Figure 8. Curve 8a shows a small linear trend. Curve 8b shows a larger linear trend. 8c shows a quadratic trend. $8 \mathrm{~d}$ shows a linear trend as well as a quadratic trend.

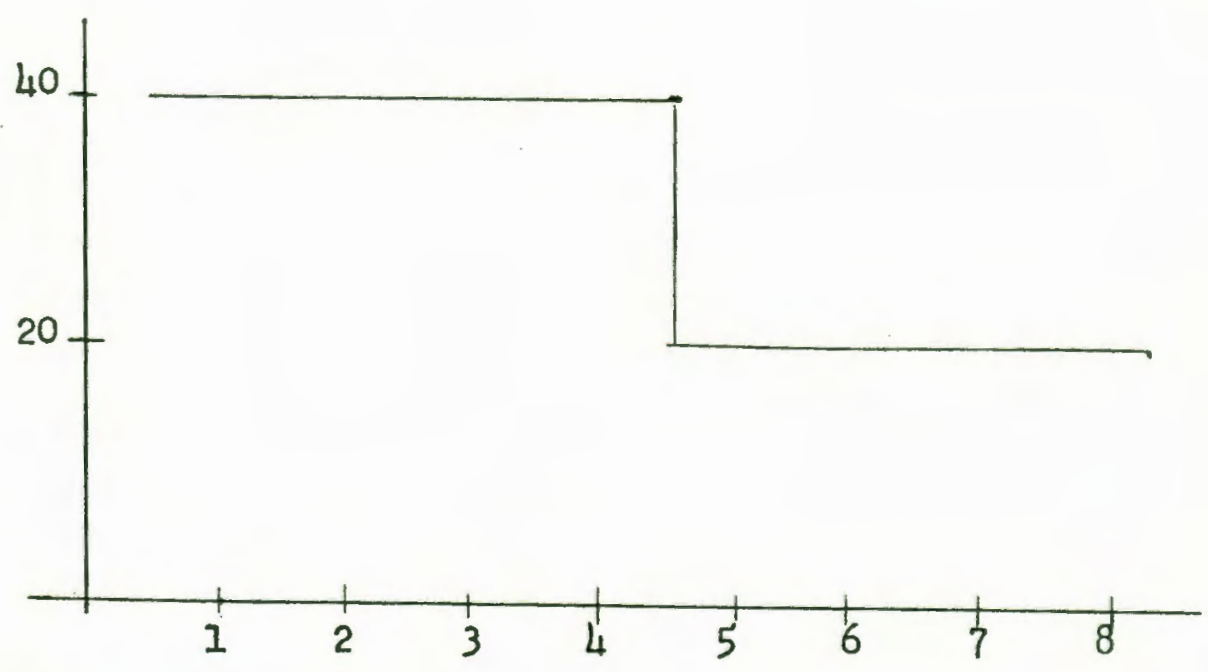

Figure 9. The first, third, and fifth polynomial trends would be significant in this simple, but non-synmetric curve. 
difficulty in interpretation of the higher trends is evidenced by the fact that tables do not usually give coefficients beyond the fifth. Even stopping at the fifth it would be difficult to guess that the curve had the given shape from the knowledge that the first, third, and fifth components rere significant. Using orthogonal polynomials for this example does not clarify the shape of the curve, but rather confuses the is sue.

\section{HAAR FUNCTIONS}

Orthogonal polynomials are not, however, the only set of orthogonal functions which can be used to analyze a curve into its components. For example, Fourier analysis is a technique for analyzing periodic functions into simple trigonometric components. Another set of orthogonal functions which are easy to use computationally and also easy to interpret are Haar functions (Harmuth, 1972).

Haar functions are step functions which take on one of three values - I, -1 , and 0 - at any value of the independent variable. Figure 10a through lod show the first four Haar functions. The first Haar function is a constant function having the value one at every point. The second Haar function equals one for the first half and minus one for the second half. A curve which had a positive component of the second Haar function would be higher in the first half than in the second. See figure Ila through Ilc.

The third Haar function is one for the first quarter of the curve, mims one for the second quarter and zero for the second half. A curve having a positive component of the thind Haar function would be higher in its first quarter than the second. 

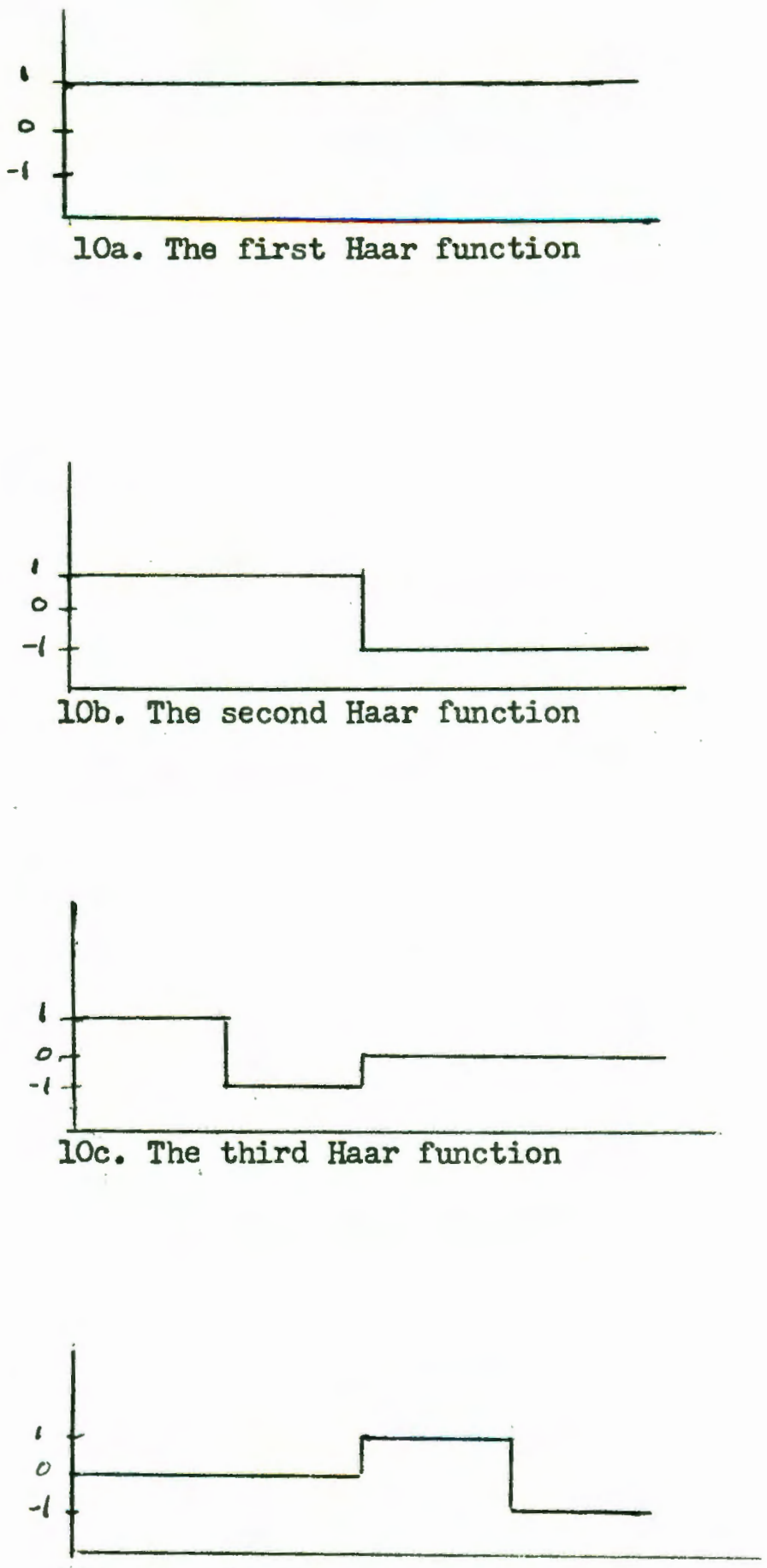

10d. The fourth Haar function

Figure 10. The first four Haar functions. 


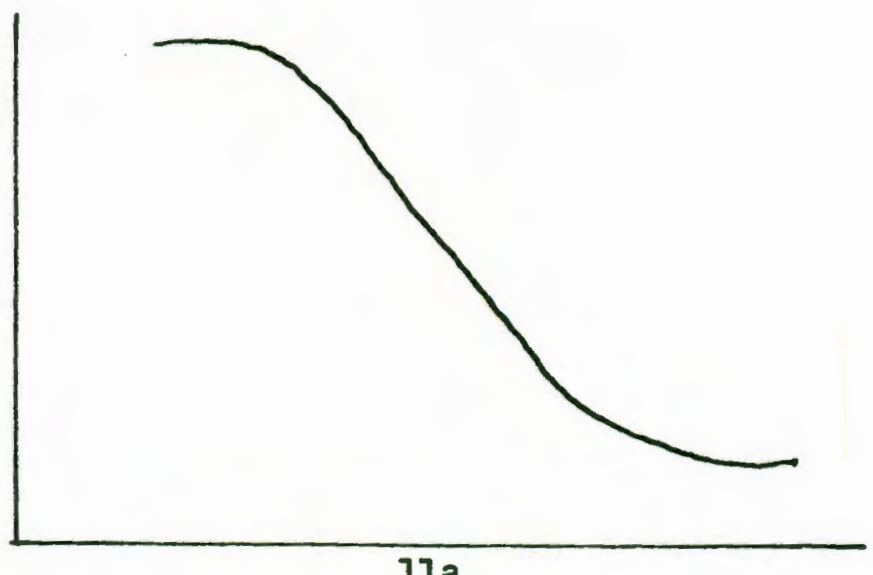

$11 a$

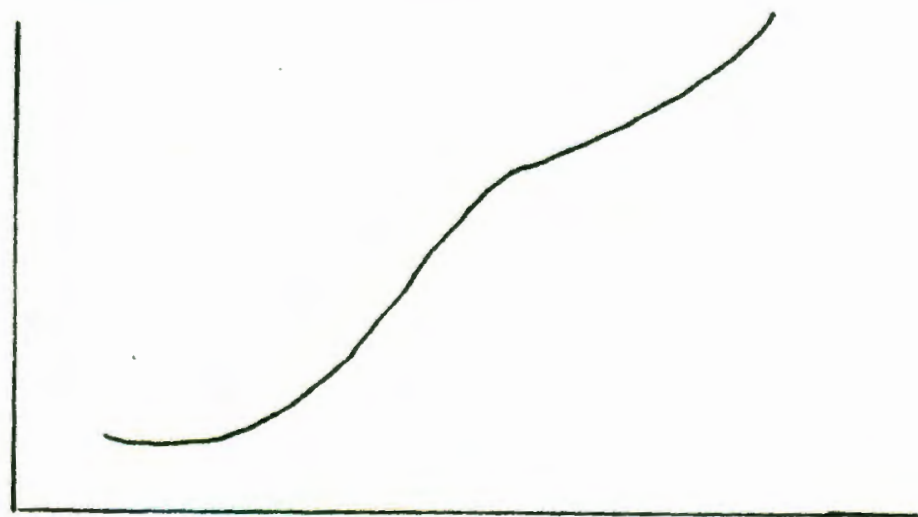

$11 b$

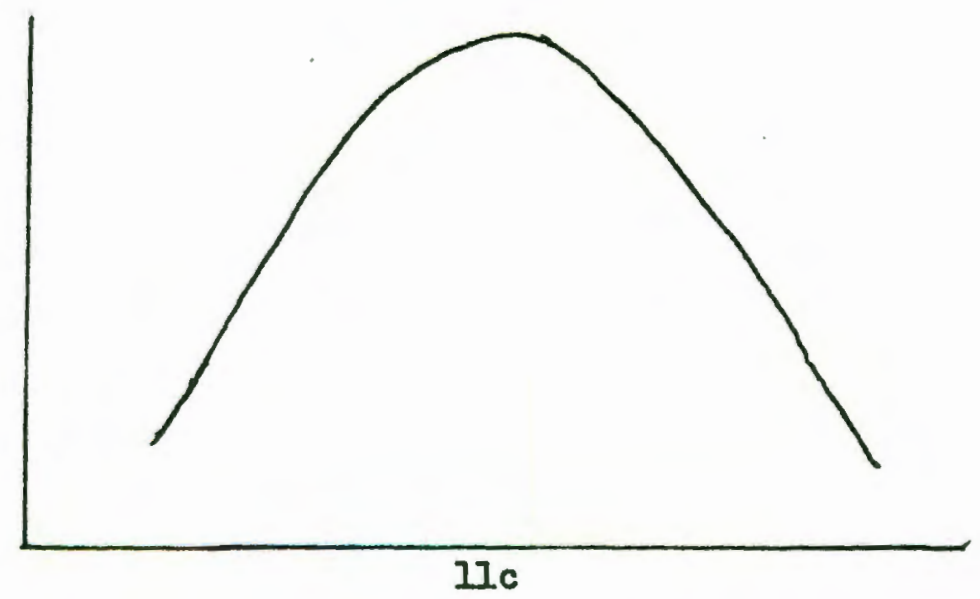

Figure 11. Figure 1la would show a significant positive component of the second Haar function, while IIb would show a significant negative component. Curve Ilc wovld not show a significant component of the second Haar function since it is the same height in both halves on the average. 
The shape of the second half of the curve does not influence whether or not this third component is significant. See figure $12 \mathrm{a}$ and $12 \mathrm{~b}$.

The fourth Haar function is equal to zero for the first half, one for the third quarter and minus one for the fourth quarter. A curve having a significant positive component of the fourth Haar function would have a third quarter which is higher than the fourth. The fifth through eighth Haar functions are equal to one for one-eighth, mims one for the adjacent one-eighth, and zero for the rest of the curve. Qurves which have a significant component of the fifth Haar function have a first eighth higher than the second eighth; a significant sixth Haar function implies a third eighth which is higher than the fourth eighth; and so on. The ninth through sixteenth Haar functions are used to determine if adjacent sixteenths of the curve differ in height. Similarly, the 17th through 32 nd Haar functions are used to determine if neighboring thirty-seconds are different in height.

To determine which Haar components are significant one must take the Innear combination of the appropriate Haar coefficients with the co-ordinate means:

$$
\psi_{h_{i}}=\underline{h}_{i}^{\prime} \overline{\mathbf{y}}
$$

where $\underline{h}_{i}$ is the set of Haar coefficients for the ith component. The Haar coefficients are not tabled in statistical text, but can easily be determined. Table VII. shows the har coefficients to use for a curve comprised of four points, eight points, and sixteen points. For a number of points equal to $2^{n}$ the coefficients can be determined analogously. 

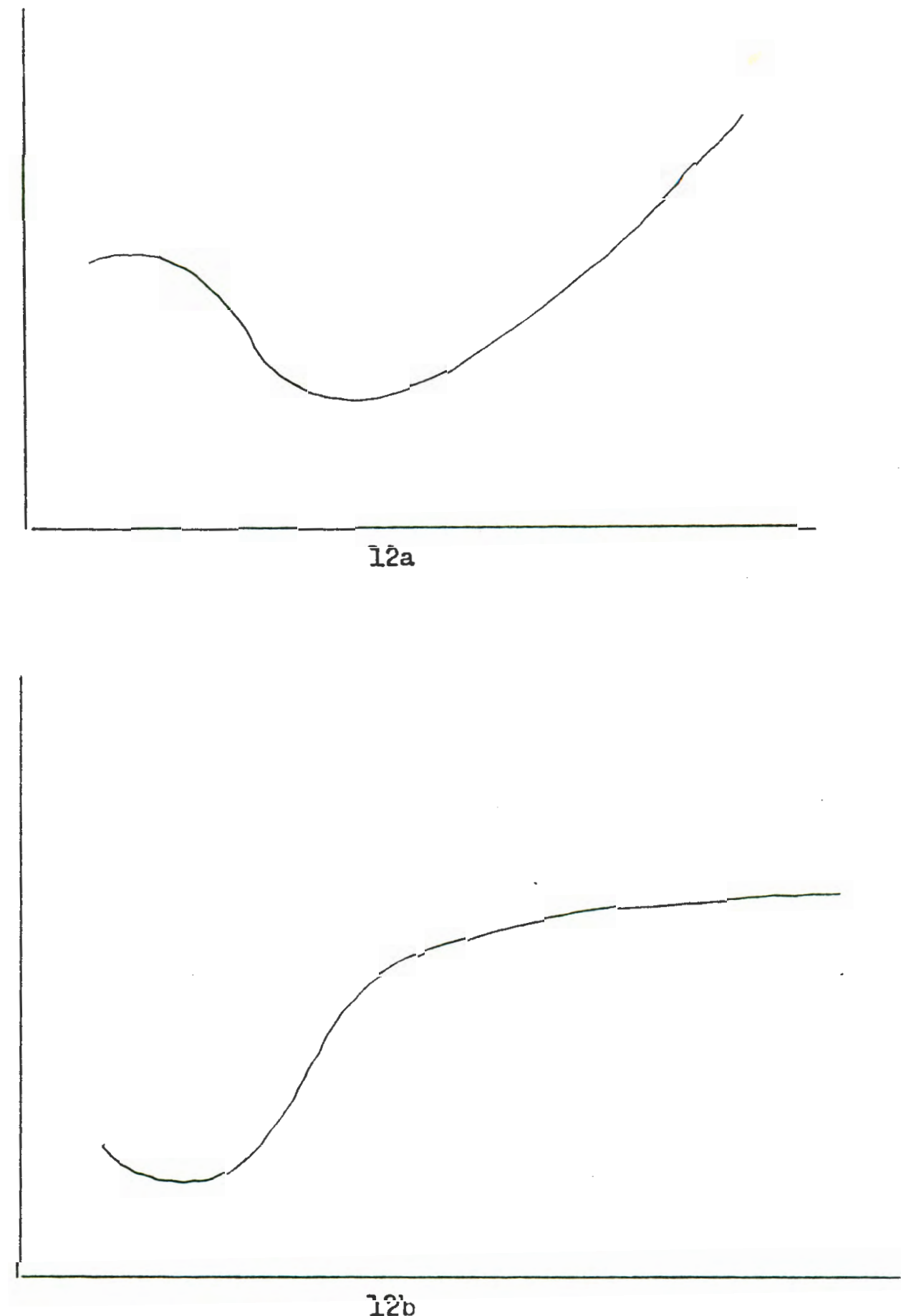

Figure 12. Gurve 12a would have a significant positive component of the third Haar function. Surve $12 b$ would have a significant negative component of the third Haar function. 


\section{TABLE VII}

HAAR COEFFICIENTS FOR OURVES COMPRISED

OF FOUR, EIGHT, OR SIXTEEN POINTS

$$
\begin{aligned}
& \begin{array}{ll}
\text { point } \\
2 \quad 3 \quad 4 \\
\hline
\end{array} \\
& \text { b. I I I I } \\
& \begin{array}{cccccc}
\underset{5}{5} & h_{2} & 1 & 1 & -1 & -1 \\
0 \\
\stackrel{9}{9} & h_{3} & 1 & -1 & 0 & 0
\end{array} \\
& \mathrm{~h}_{4} 0 \quad 0 \quad 1 \quad-1
\end{aligned}
$$

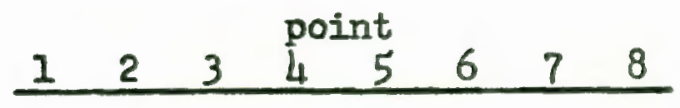

$$
\begin{aligned}
& \begin{array}{lllllllll}
\mathrm{h}_{1} & 1 & 1 & 1 & 1 & 1 & 1 & 1 & 1
\end{array} \\
& \begin{array}{lllllllll}
\mathrm{h}_{2} & 1 & 1 & 1 & 1 & -1 & -1 & -1 & -1
\end{array} \\
& \begin{array}{lllllllll}
h_{3} & I & 1 & -1 & -1 & 0 & 0 & 0 & 0
\end{array} \\
& \begin{array}{cccccccccc}
\stackrel{5}{5} & h_{4} & 0 & 0 & 0 & 0 & 1 & 1 & -1 & -1 \\
\text { 总 } & h_{5} 1 & -1 & 0 & 0 & 0 & 0 & 0 & 0
\end{array} \\
& \begin{array}{lllllllll}
h_{6} & 0 & 0 & 1 & -1 & 0 & 0 & 0 & 0
\end{array}
\end{aligned}
$$

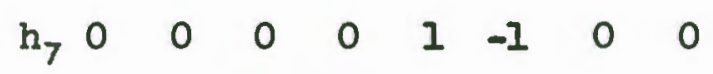

$$
\begin{aligned}
& \begin{array}{lllllllll}
h_{g} & 0 & 0 & 0 & 0 & 0 & 0 & 1 & -1
\end{array}
\end{aligned}
$$

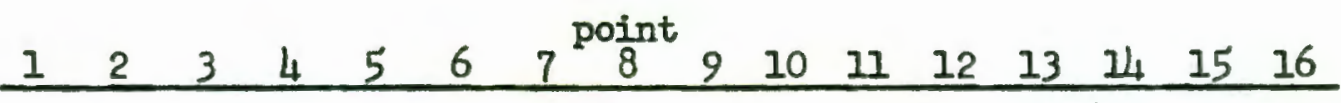


If the number of points does not equal $2^{n}$, the Haar coefficients may be determined as follows. The first function is represented by all ones, as before. Suppose that $2^{k}$ is the largest power of 2 which is less than $p$, the number of points. The second Haar function is one for the first $2^{k}$ points and minus one for the remaining $p-2^{k}$ points. The coefficients for comparing the mean levels in the two segments are $\mathrm{p}-2^{k}$ for the first $2^{k}$ points and $-\left(2^{k}\right)$ for the remainder, reflecting the unequal number of points in the two portions of the curve. Coefficients for subsequent functions within the segment with $2^{k}$ points (the first segment) can be determined in the usual way. If $\mathrm{p}-2^{\mathrm{k}}$ is an integral power of 2 (for example, if $p=12, p-2^{k}=4$ ), then coefficients for functions within this second segment can also be determined in the usual way. If not, repeat the process described above for the segment of $p-2^{k}$ points, finding the largest power of 2 which is less than $p-2^{k}$. For example, if $p=7$, the coefficients would be as shown in table VIII.

After finding the linear combination of the Haar components with the co-ordinate means, the sum of squares for the linear combination will be found and this value divided by the mean squares for error. As a numerical example, consider again the curve used in the discussion of orthogonal polynorials, where $\bar{Z}=(40,40,40,40,20,20,20,20)$ and where MS error $=40$. (See figure 9.) The sum of squares for the eight components and $\operatorname{SS}\left(\psi_{h_{i}}\right) /$ MS error is given in table IX. Comparing the value of the ratios SS/MS to the F.os value of 3.98 , only components $I$ and 2 would be significant. Finding $\psi_{n_{i}} /\left(\underline{h}_{i}^{\prime} \underline{h}_{i}\right)$ will give the magnitude and direction of 
TABLE VIII

HAAR COEFFICIENTS FOR A CURVE COMPRTSED OF SEVEN POINIS

\begin{tabular}{|c|c|c|c|c|c|c|c|}
\hline & 1 & 2 & 3 & $\begin{array}{r}\text { point } \\
\end{array}$ & 5 & 6 & 7 \\
\hline $\mathrm{h}$ & 1 & 1 & 1 & 1 & 1 & 1 & $I$ \\
\hline $\mathrm{h}$ & 3 & 3 & 3 & 3 & -4 & -4 & -4 \\
\hline$h$ & 1 & 1 & -1 & -1 & 0 & 0 & 0 \\
\hline $\mathrm{h}$ & 0 & 0 & 0 & 0 & 1 & 1 & -2 \\
\hline $\mathrm{h}$ & 1 & -1 & 0 & 0 & 0 & 0 & 0 \\
\hline $\mathrm{h}$ & 0 & 0 & 1 & -1 & 0 & 0 & 0 \\
\hline $\mathrm{h}$ & 0 & 0 & 0 & 0 & 1 & -1 & 0 \\
\hline
\end{tabular}




\section{TABTLE IX}

SUM OF SQUARES FOR HAAR COMPONENTS OF CORVE IN FIGUPE 9

\begin{tabular}{|c|c|c|}
\hline & $\operatorname{ss}\left(\psi_{h_{i}}\right)$ & $\frac{\operatorname{ss}\left(Y_{h i}\right)}{\text { MS error }}$ \\
\hline h, & $(1 \cdot 40+1 \cdot 40+1 \cdot 40+1 \cdot 40+1 \cdot 20+1 \cdot 20+1 \cdot 20+1 \cdot 20)^{2}=7200$ & 180 \\
\hline$h_{2}$ & $(I \cdot 40+1 \cdot 40+1 \cdot 40+1 \cdot 40-1 \cdot 20-1 \cdot 20-1 \cdot 20-I \cdot 20)^{2}=800$ & 20 \\
\hline$h_{3}$ & $(1 \cdot 40+1 \cdot 40-1 \cdot 40-1 \cdot 40+0 \cdot 20+0 \cdot 20+0 \cdot 20+0-20)^{2}=$ & 0 \\
\hline$h_{4}$ & $(0 \cdot 40+0 \cdot 40+0 \cdot 40+0 \cdot 40+1 \cdot 20+1-20-1 \cdot 20-1-20)^{2}=$ & 0 \\
\hline$h_{5}$ & $(1 \cdot 40-1 \cdot 40+0 \cdot 40+0 \cdot 40+0 \cdot 20+0 \cdot 20+0 \cdot 20+0 \cdot 20)^{2}=$ & 0 \\
\hline$h_{\ell}$ & $(0 \cdot 40+0 \cdot 40+1 \cdot 40-1 \cdot 40+0 \cdot 20+0 \cdot 20+0 \cdot 20+0 \cdot 20)^{2}=$ & 0 \\
\hline$h_{7}$ & $(0 \cdot 40+0 \cdot 40+0 \cdot 40+0 \cdot 40+1 \cdot 20-1 \cdot 20+0-20+0-20)^{2}=$ & 0 \\
\hline$h_{B}$ & $(0 \cdot 40+0 \cdot 40+0 \cdot 40+0 \cdot 40+0 \cdot 20+0 \cdot 20+1 \cdot 20-1-20)^{2}=$ & 0 \\
\hline
\end{tabular}


the significant components. $\psi_{h} / 8$ gives the overall level of the curve which is 30 . $\psi_{\mathrm{h}_{2} / 8}=10$, being positive indicates that the first half is higher than the second, and that on the average, the first half is ten units above the mean while the second half is ten units below. From the lnowledge of the significant Haar components, one could easily determine the shape of the curve. For this example, the Haar components provide a simple and more easily interpretable decomposition of the curve.

The procedure used above for determining which ortinogonal components (Haar or polynomial) are significant is the procecure outlined in most texts (Winer, 1971; Hays, 1973). However, as Hays points out, unless one is solely interested in detemining whether one particular component is significant, one should perform a simultaneous test on the components of interest. As noted in the discussion on finding a confidence band around one curve (p. 5), the probability that a set of statements will hold will be less than that of each individual statement unless the random variables (in this case linear combinations) are perfectly correlated.

In order to perform a simultameous test with an overall level of significance, one again turns to Miller for the formala. Two cases will again be considered.

\section{GASE I: COVARIANCE MATRIX UNWNONN}

For the general case with $\Sigma$ unknown, expression (II) gives Bonferroni intervals for all possible linear combination $\underline{I} \overline{\mathbf{Y}}$, $k$ of which hold simultaneously with probability > I-x: 
(II) $\underline{I}^{\prime} \mu \in \underline{I} \underline{I}^{\prime} \overline{\underline{Y}} \pm t_{n-1}^{\infty / 2 k}\left(\frac{\underline{I} \cdot S \underline{I}}{n}\right)^{1 / 2}$

where $k$ is the number of combinations under consideration.

Statement (II) is written in terms of a confidence band. It could be rewritten as a significance test:

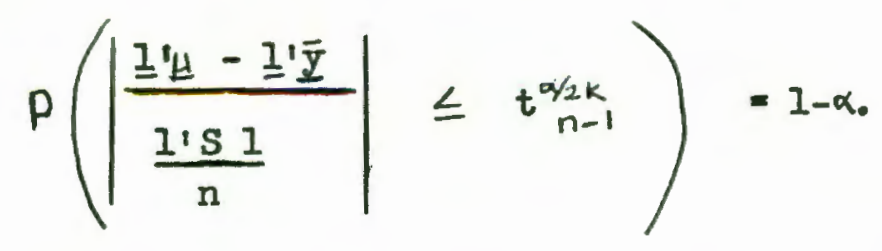

One would reject the null hypothesis that $\underline{I}^{\prime} \mu=0$ if

$$
\frac{I^{\prime} \overline{\underline{y}}}{\left(\frac{I^{\prime S I}}{n}\right)^{1 / 2}}>t_{n-1}^{\infty / 2 k}
$$

By substituting the $\underline{h}_{i}$ for the $\underline{l}$, and squaring both sides

(13) becomes:

$$
\frac{\left(\psi_{n_{i}}\right)^{2}}{h_{i}^{i} h_{i}}>\left(t_{n-1}^{2 / 2 k}\right)^{2}
$$

n

Alternatively one could use the expression

$$
P\left(\left|\frac{\underline{I} \underline{\underline{n}}-\underline{I}^{\prime} \underline{y}}{\left(\frac{n-1}{n-p}\right)^{1 / 2}\left(\frac{\underline{I}^{\prime} S}{n}\right)^{1 / 2}}\right| \leq\left(\mathrm{pF}_{p_{1} n-p}^{\alpha}\right)^{1 / 2}\right)=1-\alpha \begin{array}{r}
\forall 1 \in \mathcal{L} \text { where } \mathcal{L} \\
\text { is the set of } \\
\text { all possible linear } \\
\text { combinations, }
\end{array}
$$

which translates into the significance test:

$$
\frac{(1 \cdot \bar{z})^{2}}{\left(\frac{n-1}{n-p}\right)\left(\frac{1 ! S I}{n}\right)}=\frac{\left(\psi_{h_{i}}\right)^{2}}{\left(\frac{n-1}{n-p}\right)\left(\frac{n^{\prime} S h_{i}}{n}\right)}>\mathrm{pF}_{p, n-p}^{a} .
$$

One would reject the null hypothesis if the left side is greater than the right. Once again the value $\left(t_{n-1}^{a / 2 k}\right)^{2}$ will usually be less than 
than $\left(\frac{n-1}{n-p}\right)\left(\mathrm{pF}^{\mu}, n-\rho\right)$ unless $k$ is very large.

As a numerical example from actual data consider again the p-wave shown in figure $I$ with mean and variance shown in table $I$. Table $X$ shows $\psi_{h_{i}}, \frac{\left(\psi_{m_{i}}\right)^{2}}{\frac{h^{\prime} S \underline{h}}{n}}, \Sigma c_{i}^{2}$, and $\psi_{h_{i}} / \Sigma c_{i}^{2}$ values for this curve. With $\alpha=.01$,

$$
\left(t \frac{464}{49}\right)^{2}=15.13
$$

Using expression (14), significant components were determined, and are shown with an asterisk $(*)$ in table $X$.

In order to construct the shape of the curve from knowledge of the significant Haar components, we will look at the $4 \mathrm{hi} / 0^{2}$ values for these components. First for $\psi_{h_{1}}$, the overall level is found to be 172.85 . $\psi_{h_{1}} / 2 c_{i}^{2}=5.42$ indicating that the first half is on the average 5.42 units above the average while the second half is 5.42 units below the average.

Since there are so many components we will look at quarters of the curve separately. $\quad \psi_{h_{3}} / z c_{i}^{2}=-5.09$, indicating that the first quarter is lower tinan the second quarter. Within the first quarter, the first eighth is $2.85\left(\frac{\psi_{h i}}{\Sigma c_{i}^{2}}=-2.85\right)$ units lower than the average for that quarter, while the second eighth is 2.85 units above indicating an increase to the right in the first quarter. Breaking this into finer details, we see that the first sixteenth and the second sixteenth do not differ significantly, while the third sixteenth is 2.70 units below the average for that eighth and the fourth sixteenth is 2.70 units above $\left(4 \mathrm{mc} / 2 \mathrm{c}^{2}=-2.70\right)$. The pattern of the first eight thiry-seconds confirms the pattern of the sixteenths, with $\psi_{h_{1}}$ and $\psi_{h, g}$ not being significant while the negative values of 
$\psi_{n_{19}}$ and $\psi_{h_{20}}$ show an increasing trend to the right in the second eighth of the curve.

The second quarter was found to be 5.42 units above the average for the first half. Within this quarter, the third eighth is lower than the fourth since $\psi_{n_{6}} / 2 c_{i}^{2}=-3.68$. The comparison of the fifth sixteenth to the $\operatorname{sixth}\left(\psi_{h_{4}} / \Sigma c_{i}^{2}=.55\right)$ indicates a small decrease in level from the fifth sixteenth to the sixth, while the comparison of the seventh sixteenth to the eighth $\left(\psi_{m_{i} /} / \Sigma_{c_{i}}^{2}=-5.23\right)$ indicates that the curve again increases to the right in the next eighth. Comparisons between neighboring thirty-seconds show no significant difference between the ninth and tenth and between the eleventh and twelth thirty-seconds, while the values $\psi_{n w / \Sigma} c_{i}^{2}=-2.10$ and $\psi_{x} \sqrt{2} c_{i}^{2}$ $=-2.15$ reflect a generally even increase to the right in the third eighth of the curve.

Therefore in the second quarter, while the curve generally increases to the right, there is a slight downturn in the third eighth with the fourth eighth again increasing to the right.

In the third quarter, all components $\left(\psi_{n, 7}, \psi_{n, 2} \psi_{n_{14}}, \psi_{n_{25}}\right.$ $\left.\psi_{h_{16},} \psi_{h_{27}}, \psi_{n_{2 \gamma}}\right)$ are significant and positive indicating a relatively rapid decrease to the right in this quarter.

In the fourth quarter, the fact that $\psi_{\text {hg }}$ is not significant indicates that the curve is rather flat in this quarter. Within eighths, however, there is a decrease between the thirteenth and fourteenth sixteenths $\left(4 h_{t} s / 2 c_{i}^{2}-.93\right)$, while the fifteenth sixteenth is lower than than the sixteenth $\left(\Psi_{n, 4} / \Sigma c_{i}^{2}=-.45\right)$. Of the components relating to the difference between thirty-seconds, only the component 
TABLE $X$

HAAR COMPONENTS OF P-WAVE BEFORE ADMINISTRATION OF $\Delta^{4}$-THC

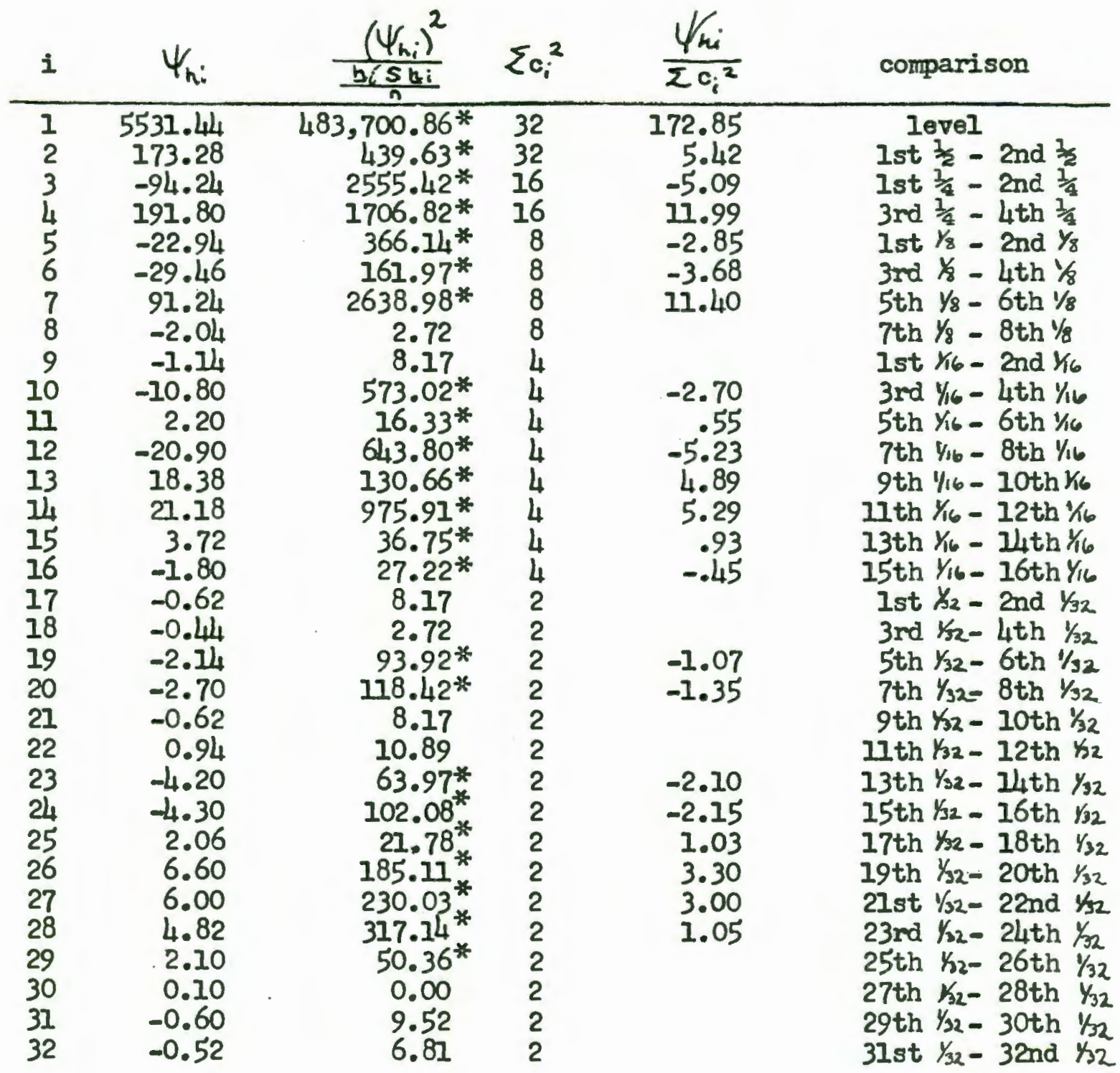


$\psi_{n \alpha q}$ is significant, indicating a decrease from the twenty-fifth thirty-second to the twenty-sixth $\left(\right.$ fhy $\left./ c^{2}=1.05\right)$.

Therefore, while the last quarter is relatively flat in comparison to the other quarters, the curve continues to decrease in the seventh eighth, but shows a flattening out and slight upturn in the last eighth.

Figure 13 shows a reconstruction of the curve from using the values of the $\psi_{h} / \sum c_{i}^{2}$ for the significant components. Also shown is the mean curve as show in figure 1. While the use of all thirtytwo Haar components can provide a very accurate fit to the curve, as in this case, the use of only eight or sixteen may give a good representation of the curve without bringing in the complications of fine divisions. However, even the highest order Haar component does lend itself to interpretation.

Using orthogonal polynomials, the following values were found for the $\psi_{F_{i}} / \frac{R_{i}^{\prime} S_{R_{i}}}{n}$ for the first five components:

$$
\begin{aligned}
& \frac{\left(\psi_{r_{1}}\right)^{2}}{\frac{p_{1}^{\prime S} \underline{p}_{1}}{n}}=1747.44 \frac{\frac{\left(\psi_{r_{2}}\right)^{2}}{\underline{p}_{2}^{\prime S} \underline{p}_{2}}}{n}=5950.77 \quad \frac{\frac{\left(\psi_{r_{3}}\right)^{2}}{\underline{\underline{p}}_{3}^{\prime S} \underline{p}_{3}}}{n}=75.47
\end{aligned}
$$

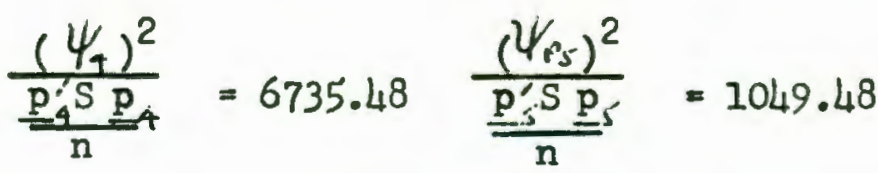

All five orthogonal components would be significant for this curve. It would be very difficult to assign physical meaning to this fact. For this example, the Haar components provide a more meaningful decomposition of this curve. 


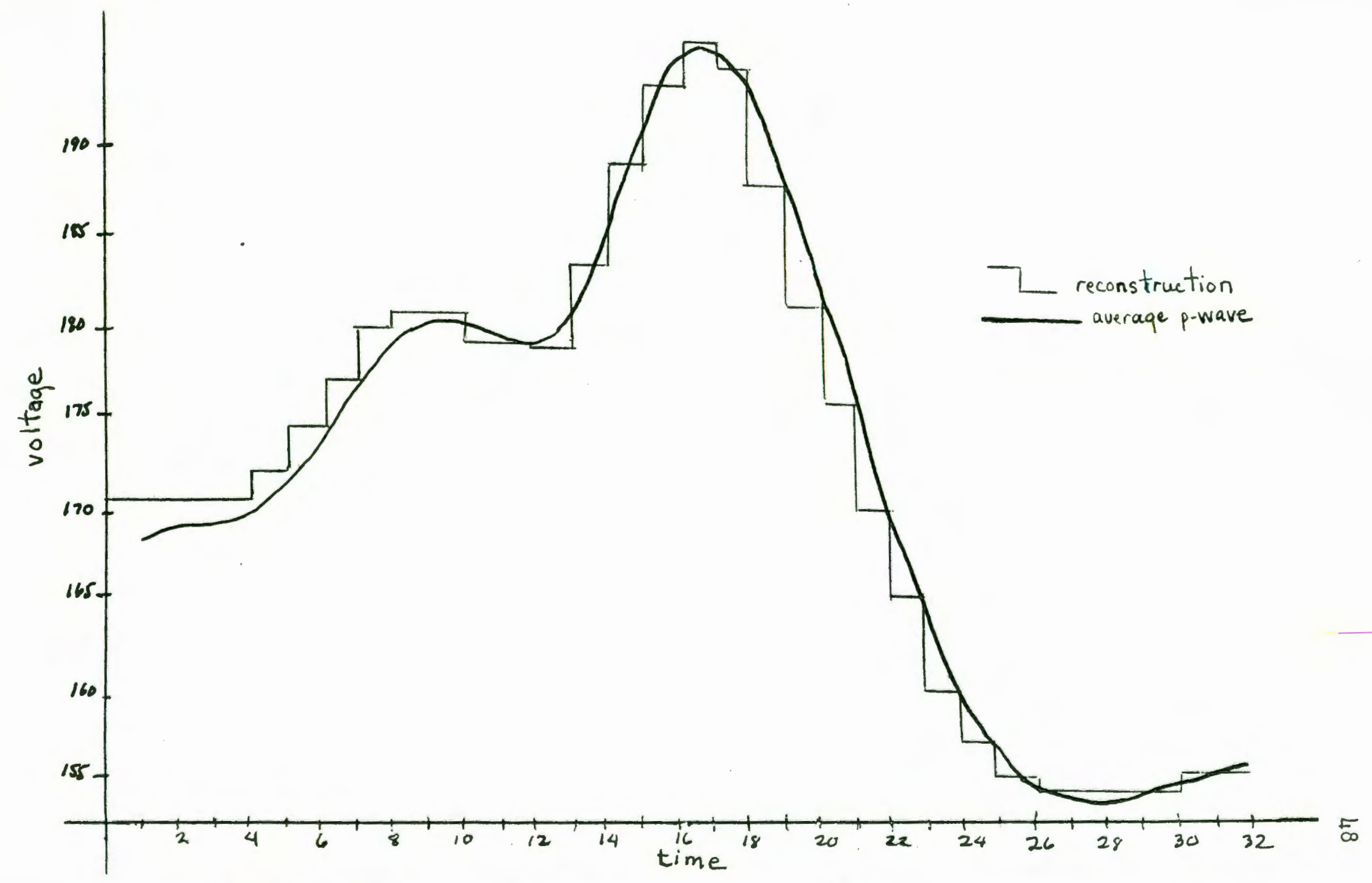

Figure 13. Average p-wave before administration of $\Delta^{9}$-THC and reconstruction of the curve from the significant haar components. 
GASE II: COVARIANGE MATRIX KNOWN EXCEPT FOR A SCATAR

In the special case where the covariance matrix is known except for a scalar $\left(\Sigma=\sigma^{2} \Sigma^{\prime}\right.$ where $\Sigma^{\prime}$ is known and $\sigma^{2}$ unknown), the formula for determining which components are significant is derived from:

$$
\underline{I}^{\prime} \mu \in \underline{I}^{\prime} \underline{\underline{Z}} \pm t_{p(n-1)}^{\alpha / 2 k}\left(\frac{s^{2} \underline{I}^{\prime}\left(\Sigma^{\prime}\right) \underline{I}}{n}\right)^{1 / 2}
$$

where $s^{2}$ is the estimate of $\sigma^{2}$ and $k$ is the number of linear combinations. Substituting the $\underline{h}_{i}$ for the 1 and re-writing in terms of a significance test this becomes -

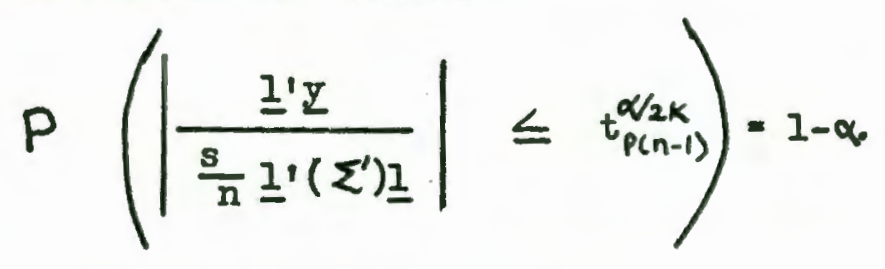

One would reject the hypothesis that $\underline{I}^{\prime} \mathcal{H}=0$ if

$$
\frac{\left(I^{\prime} y\right)^{2}}{\frac{s^{2}}{n} \underline{I}^{\prime} \Sigma^{\prime} \underline{I}}>\quad\left(t_{p(n-1)}^{\alpha / 2 k}\right)^{2}
$$

Alternatively, one could use

$$
\underline{I}^{\prime} \underline{\underline{H}} \in \underline{I}^{\prime} \underline{\underline{y}}=\left(p F_{p, p(n-1)}^{\alpha}\right)^{\frac{1}{2}}\left(\frac{s^{2}}{n} \underline{I}^{\prime} \Sigma^{\prime} \underline{I}\right)^{\frac{3}{2}}
$$

rejecting the null hypothesis if

$$
\frac{(\underline{\underline{I}} \cdot \underline{\underline{Y}})^{2}}{\frac{s^{2}}{n} \underline{I^{\prime} \Sigma^{\prime} \underline{I}}}>p F_{p, p(n-1)}^{\alpha}
$$


Once again the equation based on the Bonferroni inequality (17) will provide the better test, unless $k$ is very large.

As an example, use again the data from the experiment on the effect of drug dosage on performance of a task where independent groups were used at each level of the independent variable (p.10). Here it was assumed that the covariance matrix had the form:

$$
\Sigma=\sigma^{2}\left(\begin{array}{llll}
1 & 0 & 0 & 0 \\
0 & 1 & 0 & 0 \\
0 & 0 & 2 & 0 \\
0 & 0 & 0 & 2
\end{array}\right)
$$

The mean vector of the curve representing the effects of the syn. $\triangle 9$-THC was found to be

$$
\overline{\underline{Y}}=(58.0,61.3,44.4,36.9)
$$

with $s^{2}=56.43$. Table XI gives the values of $Y_{h i}, \underline{h} \Sigma^{\prime} \underline{h}$, and $\left(\psi_{h_{i}}\right)^{2} /\left(\frac{s^{2}}{n} h^{\prime} \Sigma^{\prime} \underline{h}\right)$ for this example. With $p=4, \alpha=.05$, $n=10$, and $k=4$,

$$
\left(t_{p(n-1)}^{\alpha / 2 k}\right)^{2}=6.92
$$

Using expression 19, the first and second Haar components are significant, while the third and fourth would not be significant. Calculating $\psi_{h a /} / \mathrm{c}_{i j}^{2}$ and $\psi_{h \mathrm{~h}} / 2 \mathrm{c}_{2, j}^{2}$ gives:

$$
\frac{\psi_{h_{1}}}{\sum_{i=1}^{L} c_{i j}}=\frac{200.6}{4}=50.12 \quad \frac{\psi_{A_{2}}}{\sum_{j=0} c_{3 j}}=\frac{38.0}{4}=9.50
$$

This shows that the overall level is 50.12 and that the first half is 9.5 units above this average while the second half is 9.5 units below 
50.12. The fact that the third and fourth components were not significant would indicate that the difference between the first quarter and the second and between the third quarter and fourth quarter were not significant. A reconstruction of the curve from this information would appear as in figure 14. Also shown is the mean curve. Using orthogonal polynomials for this example would give the values shown in table XII for $\psi_{f_{i}}, p_{i}^{\prime} L_{p}^{\prime}$ and $\left(\psi_{p_{i}}\right)^{2} / \frac{s^{2}}{n} p_{i}^{\prime} \Sigma_{p_{i}}^{\prime}$. Using expression 19 to determine which components are significant, only the first or linear component exceeds the $t$ value. Since

$$
\frac{\psi p_{i}}{\sum c_{i}^{2}}=-20.2
$$

is negative, this indicates that the curve has a downward linear trend from left to right.

For this example, the orthogonal polynomials provide at least as good if not simpler characterisation of the curve as that provided by the Haar functions. This will be the case when the curve can be closely fitted by a simple combination of polynomials, such as by a Iinear function, a quadratic function or a combination of the two. 
TABLE XI

HAAR COMPONENTS OF SWN, $\triangle^{9}-$ THC

PERPORMANCE CURVE

\begin{tabular}{|c|c|c|c|c|}
\hline$i$ & $T_{h_{i}}$ & $\underline{h} \Sigma^{\prime} \underline{h}_{i}$ & $\frac{1}{s^{2} / n h^{\prime} \Sigma^{\prime} y_{i}}$ & comparison \\
\hline 1 & 200.6 & 6 & $1188.5 *$ & level \\
\hline 2 & 38.0 & 6 & $42.68^{*}$ & Ist $\frac{1}{2}-2$ nd $\frac{1}{x}$ \\
\hline 3 & -3.3 & 2 & .96 & Ist $\frac{1}{4}-$ and $\frac{1}{4}$ \\
\hline 4 & 7.5 & 4 & 2.49 & $3 r d \frac{1}{4}-4 \operatorname{th} \frac{3}{4}$ \\
\hline
\end{tabular}

TABLE XII

POLINOMIAL COMPONENTS OF SZN. $\triangle^{9}-$ THC PERFOPMANCE CURVE

\begin{tabular}{|c|c|c|c|c|}
\hline a & $\Psi_{p i}$ & $\mathrm{p}_{i}^{\prime} \Sigma^{\prime} \mathrm{p}_{i}$ & syn $f_{i}^{i} \Sigma^{\prime} f_{i}$ & trend \\
\hline 1 & -80.2 & 30 & $37.82^{*}$ & Iinear \\
\hline 2 & -10.8 & 6 & -1.89 & quadratic \\
\hline 3 & 29.6 & 30 & 2.27 & cubic \\
\hline
\end{tabular}




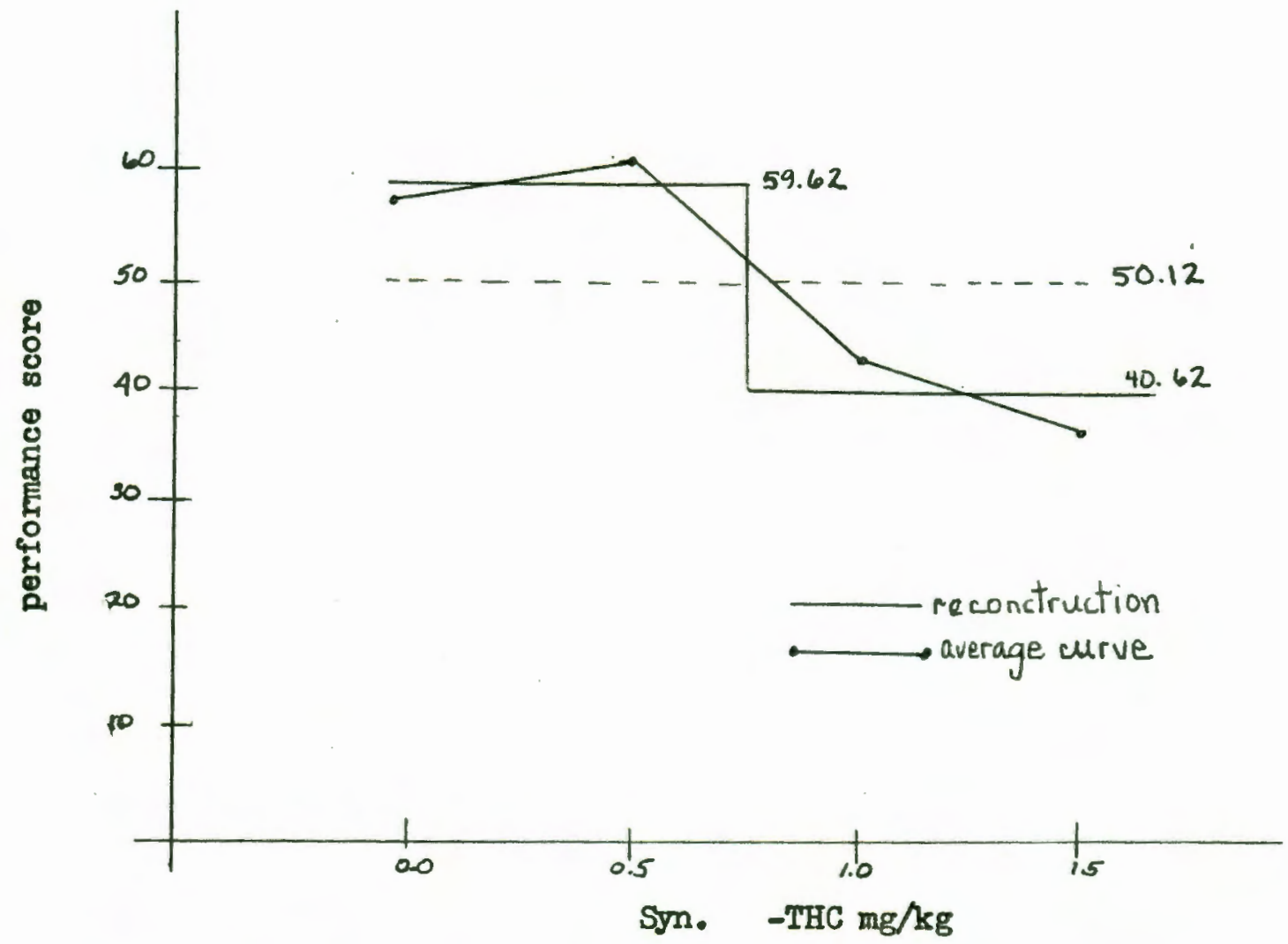

Flgure 14. Curve of average performance scores under syn. $\triangle^{4}-T H C$ and reconstruction of the curve from the significant Haar components. 
IV. COIPONENT BI COMPONENT COIPARTSON OF TWO OURVES

A natural extension of the determination of the components of one curve is the comparison of the components of two or more curves. This is a useful procedure when one wishes to compare the shape of two curves. It may be, for example, that two curves differ greatly in their overall level but have similar shapes as in figure 15. A confidence band for the difference between the two curves would indicate that there is a significant difference between the two curves at each point, but would not indicate that the two curves were of similar shape. Or, perhaps one would like to say that the first half of two curves are the same shape, but after that diverge as in figure 16.

The method presently used for making this kind of comparison would be comparing the polynomial components of the curves. However, the difficulties presented in interpreting the polynomial components of one curve are only compounded when comparing the components from two or more curves. If one or more of the curves had components beyond the first and second which are significant, comparing the Haar components rather than polynomial corponents may provide a more meaningful analysis.

Whether using Haar components or orthogonal polynomial components making a component by component comparison involves taking the linear combination of co-ordinate means of one curve and subtracting it from the same linear combination of the co-ordinate 

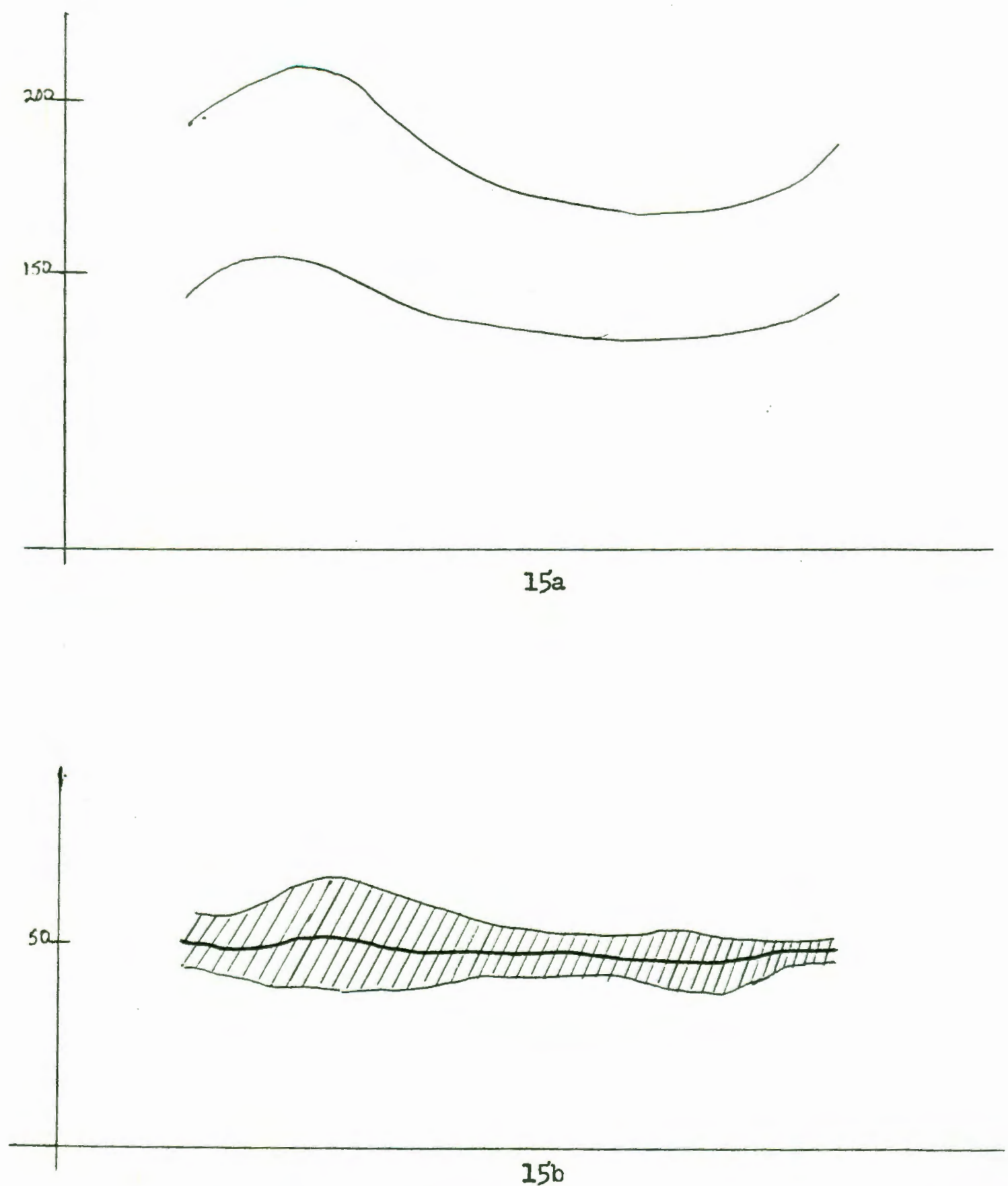

Figure 15. The curves in 15a are of similar shape but different Ieve1. I5b shows the confidence band for the difference between the curves in $15 \mathrm{a}$ with a significant difference at each point. 


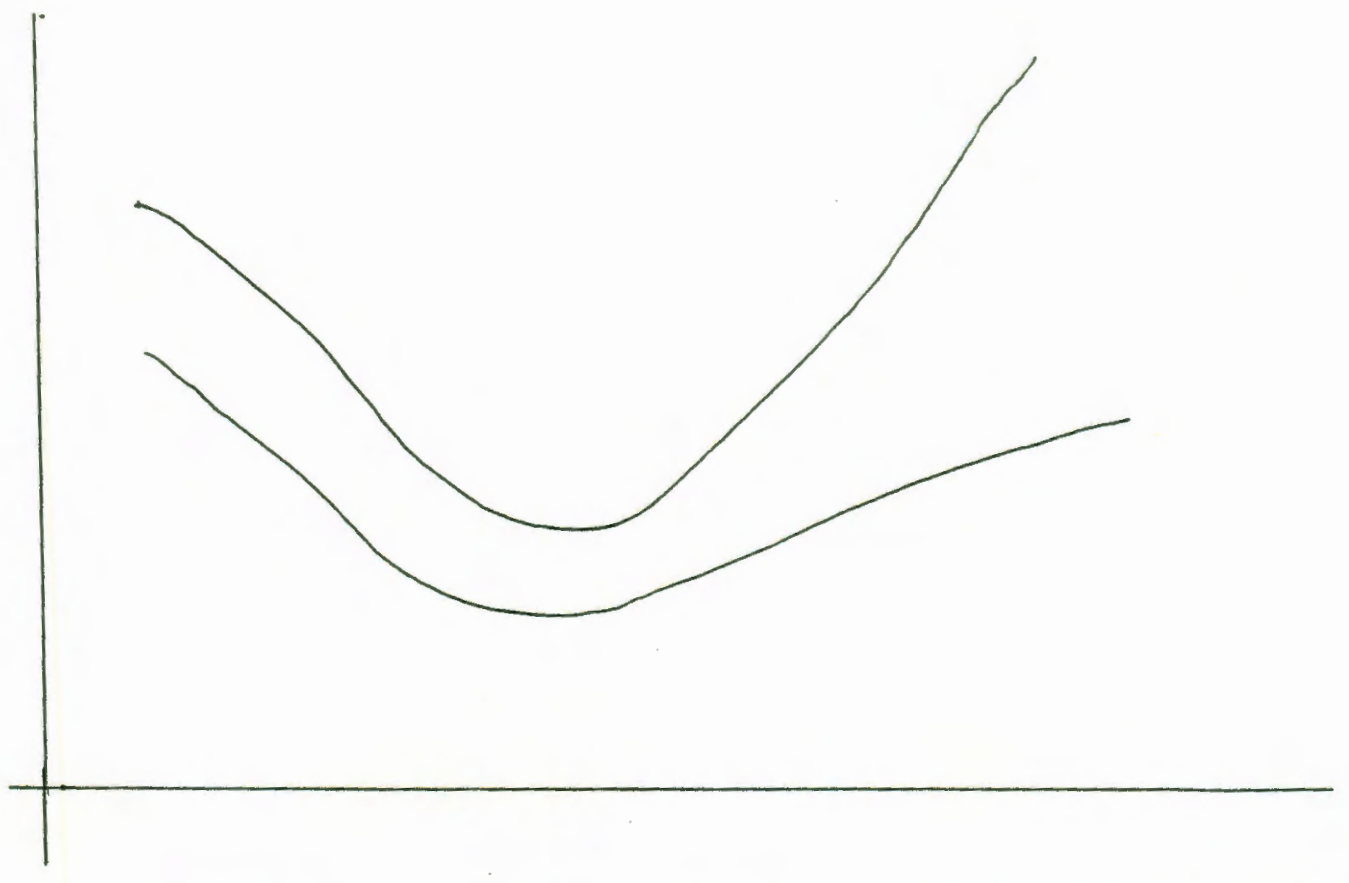

Figure 16. Curves with the same shape in the first half but with divergent shapes in the second half. 
means of the second curve. A statistical test is then made of this difference by dividing it by a factor of the covariance matrix and comparing this to a test statistic.

\section{CASE I: COTARTANCE MATRIX UNKNOWN}

For this case with unknown, the folloring expression holds:

(22)

$$
P\left(\left|\frac{\left(\underline{I}^{\prime} \mu_{1}-\underline{I}^{\prime} \mu_{2}\right)-\left(\underline{I}^{\prime} \overline{\underline{Z}}_{1}-\underline{I}^{\prime} \bar{Z}_{2}\right)}{\left(\frac{2 \underline{I}^{\prime} s \underline{I}}{n}\right)^{1 / 2}}\right| \leq t_{2(n-1)}^{\alpha / 2 x^{\prime}}\right)=1-\alpha_{0}
$$

where $S$ is the pooled estimate of the covariance matrix and where $k$ is the number of components being compared. One would reject the mull hypothesis that $\underline{I}^{\prime} \overline{\bar{y}}-\underline{\underline{I}}^{\prime} \overline{\mathrm{L}}_{2}=0$ if

$$
\frac{I^{\prime} \Psi_{1}-I^{\prime} E_{2}}{\left(\frac{I^{\prime} S I}{n}\right)^{1 / 2}}>t_{2(n-1)}^{\alpha / 2 . k}
$$

For example, consider the case of the two p-waves show in figure 2 (p. 20). The difference between respective Haar components was calcaulated. Table XIII gives values of $\underline{h}_{i}^{\prime} \overline{\bar{u}}, \underline{h}_{i}\left(\overline{\mathbf{I}}_{2},\left(\underline{h}_{i} \cdot \overline{\mathrm{Y}}_{1}-\underline{h}_{i} \overline{\mathrm{I}}_{2}\right)\right.$, and $\frac{\frac{h_{i} \bar{I}_{1}-\underline{h}_{i}^{\prime} \bar{Z}_{2}}{2 \underline{h}_{i}^{\prime} \underline{h}_{i}}}{n}$. Using expression (23), the significant differences

were determined and are shown with an asterisk in table vIII.

Since the difference between the first Haar cormonent in each curve was significant, the two curves differed in overall level. Since the difference was positive, the first curve was higher on the average than the second. The difference between the second Haar component of each ourve was also significant, with the first curve 


\section{TABLE XIII}

CONPARISON OF HAAR CONPONENTS OF P-WAVES BEFORE AND AFTER ADMINISTRATION

$$
\text { OF } \Delta^{9} \text {-THC }
$$

\begin{tabular}{|c|c|c|c|c|}
\hline 1 & his & $\underline{h}_{1}^{i} \bar{z}_{2}$ & $\underline{\underline{h}}=\bar{z}_{1}-h_{1}^{\prime} \bar{Y}_{2}$ & $\left(\frac{2 h_{i} 5 h_{i}}{n}\right)^{1 / 2}$ \\
\hline 1 & 5531.44 & 4991.08 & 540.36 & $49.8^{*}$ \\
\hline 2 & 173.28 & -38.24 & 211.52 & $12.0^{*}$ \\
\hline 3 & -94.24 & 73.14 & -167.38 & $-23.35^{*}$ \\
\hline 4 & 191.80 & -151.82 & 343.62 & $42.19^{*}$ \\
\hline 5 & -22.94 & 1.98 & -24.92 & $-12.60^{*}$ \\
\hline 6 & -20.46 & 37.28 & -66.74 & $-16.29^{*}$ \\
\hline 7 & 91.24 & -37.30 & 128.54 & $32.99^{*}$ \\
\hline 8 & -2.04 & -24.20 & 22.16 & $7.30^{*}$ \\
\hline
\end{tabular}

*significant at .01 level 
showing a greater difference in level between first half and second half than is evident in the second curve. In fact, as can be seen from the values of $\frac{h}{-2} \overline{z_{1}}$ and $\underline{h}_{-2} \bar{z}_{2}$, the difference is one of direction as well as size, with the first curve having a higher first half and the second curve a higher second half.

The difference between the third Haar components is also significant, the first curve having a negative value for $\underline{h}_{-3}^{8} \bar{z}$, while the second curve has a positive value. Hence, the first curve rises from the first quarter to the second, while the second curve decreases. Iooking at the difference between the fourth Haar components, one sees that there is also a significent difference here with the first curve showing a decrease from the third quarter to the fourth and the second curve showing an increase from third quarter to fourth.

From the analysis so far, it can be seen that these two curves differ significantly in level as well as shape. Curve 2 seems to be a rough reversal of curve 1 with curve 1, however, showing a greater difference in level between first half and second half than is evident in curve 2.

Analysis of the remaining differences in components will add more detail to the difference in shape. One notes that curve 1 shows a greater change in level from the first eighth to the second than is shown in curve 2. Gurve 2 remains rather flat in this region $\left(h_{-}^{\prime} \bar{I}_{2}=1.98\right)$. Differences in the siath and seventh components reflect the reversed trends in the two curves mentioned earlier. Differences in the eighth components of the two curves indicate that the second curve shows a much greater change in 
level from the seventh eighth to the eighth eighth than that show in curve 2.

These two curves differed markedly in shape with all of the eight components which were compared showing significant differences. The confidence band for the difference between the two curves (see figure 3) showed a difference at all bat two points. Doing the component by component analysis of these two curves allows one to say more about how the two curves differ. For this experiment, this analysis of the components allows one to say that the administration of $\triangle 9$-THC lowered the overall level of the p-isave and actualiy effected a reversal in the shape of the curve. 
CASE II: COVARIANCE MATRIX KNOWN EXCEPT FOR A SCALAR

For this case, with $\Sigma=c^{2} \Sigma^{\prime}$, the following expression is true:

(24)

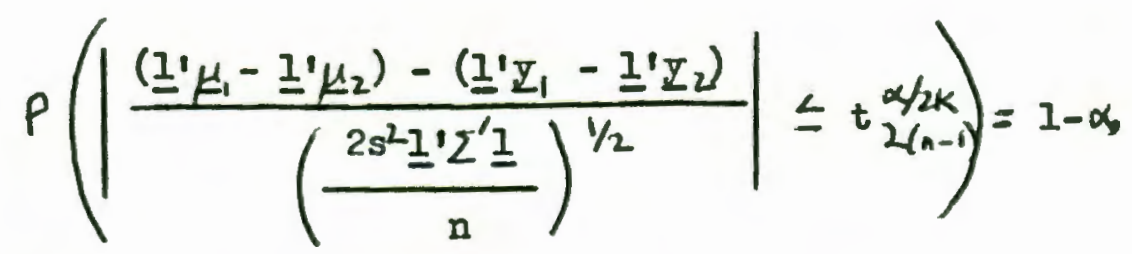

where $s^{2}$ is the pooled estimate of $\sigma^{2}$. One would reject the null hypothesis that $\left(\underline{I}^{\prime} \mu_{1}-\underline{I}^{\prime} \mu^{\prime}\right)=0$ if

$$
\frac{I^{\prime} z_{1}-I^{\prime} z_{2}}{\left(\frac{2 s^{2} I^{\prime} Z^{\prime} I}{n}\right)^{1 / 2}}>t_{2(n-1)}^{\alpha / 2 k}
$$

For an example, wo will again use the data from the experiment on the dosage level effects on performance of a task (p. 22). The mean curve for the sym. $\Delta^{\mathfrak{g}}$-THC was found to be $y=(58.0,61.3,44.4$, 36.9) and for the $\Delta^{5}$-THC was $Y=(63.3,59.6,33.2,21.8)$. The pooled estimate of $S^{2}$ would be 53.61. Table XIV shows the value of

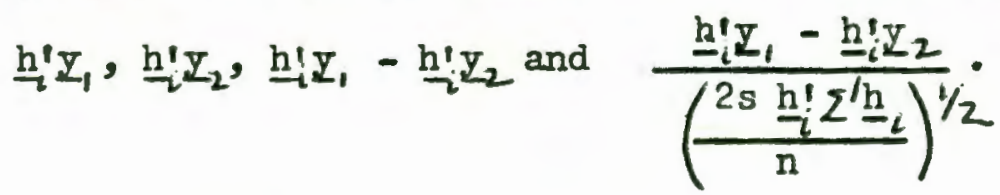

For $\alpha=.05, t_{2}^{\alpha / 8}=2.81$. Thus the difference between the first Haar components and between the second Haar components would be significant. Since,

$$
\frac{\psi_{11}-\psi_{21}}{\Sigma c_{1}^{2}}=\frac{22.7}{4}=5.67 \frac{\psi_{12}-\psi_{22}}{\Sigma c_{i}{ }^{2}}=\frac{-29.9}{4}=-7.47
$$

the overall level of the first curve is 5.67 units higher than the 


\section{TABLE XIV}

COMPARISON OF HAAR COMPONENTS OF DOSE-RESPONSE CURVES OF SIN. $\triangle$-THC AND $\Delta^{9}$-THC

\begin{tabular}{|c|c|c|c|c|c|}
\hline$i$ & $\underline{n} \underline{\underline{1}}$ & $\underline{\underline{h}} \mathbf{I}_{2}$ & 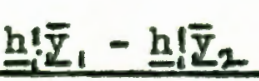 & C.mb!2 & $\left.\frac{2}{b i}\right)^{1 / 2}$ \\
\hline 1 & 200.6 & 177.9 & 22.7 & $2.83^{*}$ & \\
\hline 2 & 38.0 & 67.9 & -29.9 & $-3.73^{*}$ & , \\
\hline 3 & -3.3 & 3.7 & -7.0 & -1.51 & \\
\hline 4 & 7.5 & 11.4 & -3.9 & -.59 & \\
\hline
\end{tabular}

*significant at .05 level 
second, and the second curve showed a greater difference between the first half and the second that the first curve showed.

In an interpretation of these results with reference to this particular experiment, it could be said that the performance scores were on the average higher with the syn. $\Delta^{9}-$ THC than with $\Delta^{9}-$ THC, and that the two bigher dosage levels of the second drug effected a greater change in performance than the two higher levels of drug 1. 


\section{CONCLUSION}

The interpretaion of data from psychological experiments often involves the analysis and comparison of curves. This paper has summarized methods presented in texts on psychological statistics for the comparison of curves and has presented some alternative methods.

The first kind of analysts discussed was that of finding a confidence band around one curve. There are no methods commonly in use for finding this confidence band. It was mentioned that one might be tempted to find a confidence interval around each point using an $\propto$ level of significance for each interval. Doing so does not take into account the degree of correlation between the coordinate means and will result in an overall significance level which would be mach too low. The alternative discussed allowed one to determine simaltaneous intervals such that all of the intervals would cover their respective mean with $1-\alpha$ probability.

The second kind of analysis discussed was finding a confidence band around the difference botween two curves. Current methods presented were visual inspection, analysis of variance, and.

Hotelling's $T^{2}$. Visual inspection of curves presents the same dirficulty as visual inspection of single values, in that variance and covariances may be so large that observed differences would not be statistically significant. Visual analysis can however point out obvious differences in different portions of the curve which would be obscurred by the global analysis of ANOVA or Hotelling's $\mathrm{T}^{2}$. 
Also, in the case of repeated measure, the use of ANOVA requires a prior test on the shape of the covariance matrix which involves difficult and computer time-consuming computations.

The alternative method discussed involved finding simultaneous confidence intervals about the difference between co-ordinate means. This method allows one to determine rather easily at which points two curves differ significantly and at which points the difference is not significant. This method eliminates the need for finding the determinant of the covariance matrix which is necessary for ANOVA with repeated measures.

Next discussed was finding the significant components of a curve. The best method presently discussed in texts on psychological statistics is the method of orthogonal polynomials. The problem with this is the difficulty in interpreting the meaning of significant trends beyond quadratic. When trends such as cubic and quartic and beyond are significant, it becomes difficult to visualize the shape of the curve from knowledge of the significant trends. The alternative presented was the use of Haar functions. All of the significant Haar functions may be interpreted and the shape of the curve determined no matter how many components are significant. In some cases, however, using Haar components may provide less easily interpreted results if the curve can be characterized by a simple combination of low degree polynomials.

The last type of analysis discussed was that of comparing the components of two or more curves. The comparison of orthogonal polynomial components was discussed as the method presently in use. The results of this method are difficult to interpret if one or more 
of the curves has several significant components. The comparison of Haar components may in such cases provide a more easily understood sunmarization of the differences in shape of two curves. As discussed above, the new methods can in some cases provide advantages over existing techniques. It is hoped that these new techniques will be incorporated into the analysis of psychological data. 
Box, G. E. D.: Some theorems on quadratic forms applied in the study of analysis of variance problems, Ann. Math. Statist., $1954,25,290-302,484-498$.

Harmuth, H.: Transmission of Information by Orthogonal Functions, New York: Springer - Vertag, 1972.

Hays, W. C.: Statistics for the Social Sciences, New Iork: Holt, Pinehart, and Winston, 1973.

Miller, Simultaneous Statistical Inference, New York: McGraw-Hill, 1966.

Morrison, D. F.: Multivariate Statistical Methods, New York: McGraw-Hill, 1967.

Froy, S. N. and R. C. Bose: Simultareous confidence interval estimation, Ann. Math. Statist., 1953, 24, 513-536.

Winer, B. J., Statistical Principles in Experimental Design, New York: McGraw-Hill, 1971. 


\section{APPENDIX}

The following is an explanation of the vector and matrix multiplication which is used throughout the text to simplify notation and calculation.

Let $\underline{x}$ and $\underline{z}$ be vectors. A vector as it is normally written (x) is usually considered to be a column vector, that is, with its elements arranged in a colum:

$$
\underline{x}=\left(\begin{array}{l}
x_{1} \\
x_{2} \\
x_{3} \\
x_{4}
\end{array}\right)
$$

A vector written with a prime $\left(\underline{x}^{\prime}\right)$ is a row vector:

$$
\underline{x}^{\prime}=\left(x_{1}, x_{2}, x_{3}, x_{4}\right) \text {. }
$$

By definition, a column rector can be multiplied on the left by a row vector. The product is found by multiplying corresponding elements and suming these products:

$$
\underline{x}^{\prime} y=\left(x_{1}, x_{2}, x_{3}, x_{4}\right)\left(\begin{array}{l}
y_{1} \\
y_{2} \\
y_{3} \\
y_{4}
\end{array}\right)=\begin{array}{r}
x_{1} y_{1}+x_{2} y_{2} \\
+x_{3} y_{3}+x_{4} y_{4}
\end{array}
$$

For example if $x=\left(\begin{array}{c}1 \\ 1 \\ -1 \\ -1\end{array}\right)$ and $y=\left(\begin{array}{l}36 \\ 39 \\ 43 \\ 35\end{array}\right)$,

$$
\underline{x}^{\prime} y=(1,1,-1,-1)\left(\begin{array}{l}
36 \\
49 \\
43 \\
35
\end{array}\right)=36+39-43-35=-3
$$

The multiplication of a vector times a matrix is similar. A matrix can be multiplied on the left by a row vector and on the right 
by a colum vector:

$$
\underline{x^{\prime}} \cdot S \text { or } S \cdot \underline{x}
$$

Let $S$ be a matrix such that

$$
s=\left(\begin{array}{llll}
s_{11} & s_{12} & s_{i 3} & s_{14} \\
s_{21} & s_{22} & s_{23} & s_{24} \\
s_{31} & s_{32} & s_{33} & s_{34} \\
s_{41} & s_{42} & s_{43} & s_{44}
\end{array}\right)
$$

$S$ could be considered to be made up of four colum vectors of four elements each, or four row vectors of four elements each:

$$
\begin{gathered}
s=\left(\begin{array}{l}
s_{11} \\
s_{21} \\
s_{31} \\
s_{41}
\end{array}\right)\left(\begin{array}{l}
s_{12} \\
s_{22} \\
s_{32} \\
s_{42}
\end{array}\right)\left(\begin{array}{l}
s_{13} \\
s_{23} \\
s_{33} \\
s_{43}
\end{array}\right)\left(\begin{array}{l}
s_{14} \\
s_{24} \\
s_{34} \\
s_{44}
\end{array}\right) \\
\left(s_{11} s_{12} s_{13} s_{14}\right) \\
\left(s_{21} s_{22} s_{23} s_{34}\right) \\
\left(s_{31} s_{32} s_{33} s_{34}\right) \\
\left(\begin{array}{llll}
s_{41} & s_{42} & s_{43} & s_{44}
\end{array}\right)
\end{gathered}
$$

Considering $S$ to be composed of 4 column vectors, left multiplication by a row vector consists of multiplying the row vector times each of the colums. The result is a row vector with 4 elements:

$$
\underline{x}^{\prime} \cdot s=\left(\begin{array}{llll}
x_{1} & x_{2} & x_{3} & x_{4}
\end{array}\right) \cdot\left(\left(\begin{array}{l}
s_{11} \\
s_{21} \\
s_{31} \\
s_{41}
\end{array}\right)\left(\begin{array}{l}
s_{12} \\
s_{22} \\
s_{32} \\
s_{42}
\end{array}\right)\left(\begin{array}{l}
s_{13} \\
s_{23} \\
s_{33} \\
s_{43}
\end{array}\right)\left(\begin{array}{l}
s_{14} \\
s_{24} \\
s_{34} \\
s_{14}
\end{array}\right)\right)
$$


$-\left(\begin{array}{llll}x_{1} & x_{2} & x_{3} & x_{4}\end{array}\right)\left(\begin{array}{l}s_{11} \\ s_{41} \\ s_{31} \\ s_{41}\end{array}\right),\left(x_{1} x_{2} x_{3} x_{4}\right)\left(\begin{array}{l}s_{12} \\ s_{22} \\ s_{32} \\ s_{42}\end{array}\right)$,

$$
\left.\left(x_{1} x_{2} x_{3} x_{4}\right)\left(\begin{array}{l}
s_{13} \\
s_{23} \\
s_{33} \\
s_{34}
\end{array}\right),\left(x_{1} x_{2} x_{3} x_{4}\right)\left(\begin{array}{l}
s_{14} \\
s_{24} \\
s_{34} \\
s_{44}
\end{array}\right)\right)
$$

$=\left(x_{1} s_{11}+x_{2} s_{21}+x_{3} s_{31}+x_{4} s_{41}, x_{1} s_{12}+x_{2} s_{22}+x_{3} s_{32}+x_{4} s_{42}\right.$

$\left.x_{i} s_{i 3}+x_{2} s_{23}+x_{3} s_{33}+x_{4} s_{43}, x_{14} s_{14}+x_{2} s_{24}+x_{3} s_{34}+x_{44} s_{44}\right)$

For example:

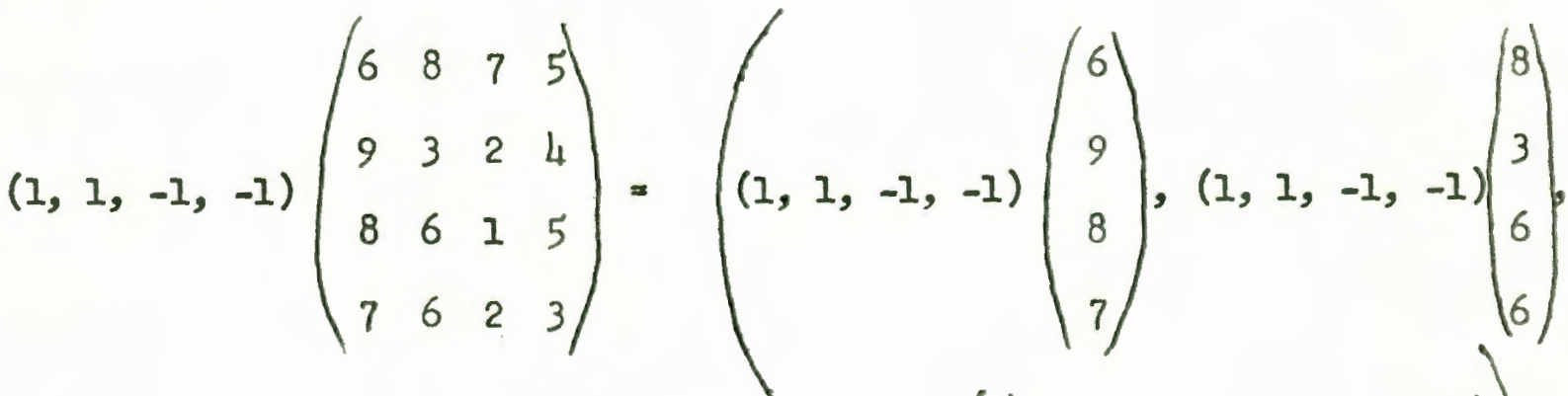

$$
\begin{aligned}
& (1,1,-1,-1)\left(\begin{array}{l}
7 \\
2 \\
1 \\
2
\end{array}\right),(1,1,-1,-1)\left(\begin{array}{l}
5 \\
4 \\
5 \\
3
\end{array}\right) \\
& =((6+9-8-7),(8+3-6-6),(7+2-1-2),(5+4-5-3)) \\
& =(2,-1,6,1)
\end{aligned}
$$

A matrix can be right multiplied by a column vector. In this 
case, consider the matrix to be composed of rows. Each row is multiplied separately times the column vector, with the result being a column vector:

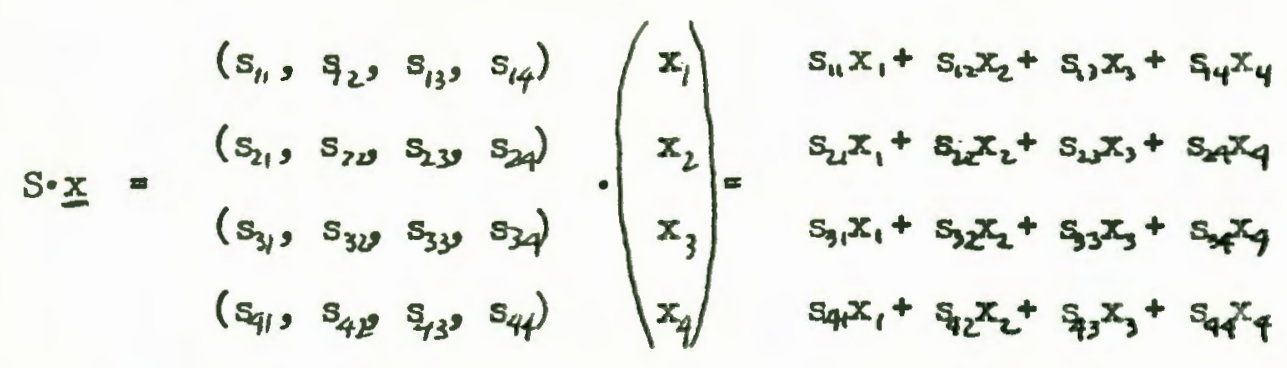

For example:

$$
\left(\begin{array}{llll}
6 & 8 & 7 & 5 \\
9 & 3 & 2 & 4 \\
8 & 6 & 1 & 5 \\
7 & 6 & 2 & 3
\end{array}\right) \cdot\left(\begin{array}{c}
1 \\
1 \\
-1 \\
-1
\end{array}\right)=\left(\begin{array}{l}
6+8-7-5 \\
9+3-2-4 \\
8+6-1-5 \\
7+6-2-3
\end{array}\right)=\left(\begin{array}{l}
2 \\
6 \\
9 \\
8
\end{array}\right)
$$

It should be noted that $x^{\prime} \cdot S$ does not necessarily equal S.x.

As an example of vector and matrix multiplication involving the Haar coefficients, consider a curve having an average curve of

$$
\bar{y}=(7,12,15,10)
$$

and covariance matrix of:

$$
S=\left(\begin{array}{cccc}
12 & 5 & 8 & 2 \\
5 & 14 & 9 & 3 \\
8 & 9 & 15 & 4 \\
2 & 3 & 4 & 6
\end{array}\right)
$$

The Iinear combinations of the Haar coefficients with the vector means 
are written as $h_{1}^{\prime} \bar{y}, h_{2} \cdot \bar{z}, h_{-3}^{\prime} \bar{z}, h_{-4}^{\prime} \bar{y}$ and would be conputed as follows:

$$
\begin{aligned}
& h_{1}^{\prime} \bar{z}=(1,1,1,1)\left(\begin{array}{l}
7 \\
12 \\
15 \\
10
\end{array}\right)=7+12+15+10=44 \\
& h_{2}^{\prime} \bar{z}=(1,1,-1,-1)\left(\begin{array}{l}
7 \\
12 \\
15 \\
10
\end{array}\right)=7+12-15-10=-6 \\
& h_{3}^{\prime} \bar{y}=(1,-1,0,0)\left(\begin{array}{l}
7 \\
12 \\
15 \\
10
\end{array}\right)=7-12+0+0=-5 \\
& h_{4}^{\prime} \bar{y}=(0,0,1,-1)\left(\begin{array}{l}
7 \\
12 \\
15 \\
10
\end{array}\right)=0+0+15-10=5
\end{aligned}
$$

h! Sh, would be found by

$$
\operatorname{hish}_{1 \rightarrow 1}=\left(\begin{array}{llll}
1 & 1 & 1 & 1
\end{array}\right)\left(\begin{array}{cccc}
12 & 5 & 8 & 2 \\
5 & 14 & 9 & 3 \\
8 & 9 & 15 & 4 \\
2 & 3 & 4 & 6
\end{array}\right)\left(\begin{array}{l}
1 \\
1 \\
1 \\
1
\end{array}\right)
$$


$=(12+5+8+2,5+14+9+3,8+9+15+4,2+3+4+6)\left(\begin{array}{l}1 \\ 1 \\ 1 \\ 1\end{array}\right)$

$=(27,31,36,15)\left(\begin{array}{l}1 \\ 1 \\ 1 \\ 1\end{array}\right)=27+31+36+15=109$

$\underline{h}_{2}^{1} \underline{S h}_{2}=(1,1,-1,-1)\left(\begin{array}{rrrr}12 & 5 & 8 & 2 \\ 5 & 14 & 9 & 3 \\ 8 & 9 & 15 & 4 \\ 2 & 3 & 4 & 6\end{array}\right)\left(\begin{array}{c}1 \\ 1 \\ -1 \\ -1\end{array}\right)$

$=(12+5-8-2,5+14-9-3,8+9-15-4,2+3-4-6)\left(\begin{array}{c}1 \\ 1 \\ -1 \\ -1\end{array}\right)$.

$=(7,7,-2,-5)\left(\begin{array}{c}1 \\ 1 \\ -1 \\ -1\end{array}\right)=7+7+2+5=21$

$$
\underline{h}_{3} \underline{s}_{3}=(1,-1,0,0)\left(\begin{array}{rrrr}
12 & 5 & 8 & 2 \\
5 & 14 & 9 & 3 \\
8 & 9 & 15 & 4 \\
2 & 3 & 4 & 6
\end{array}\right)\left(\begin{array}{c}
1 \\
-1 \\
0 \\
0
\end{array}\right)
$$




$$
\begin{aligned}
& =(12-5,5-14,8-9,2-3)\left(\begin{array}{c}
1 \\
-1 \\
0 \\
0
\end{array}\right)=(7,-9,-1,-1)\left(\begin{array}{c}
1 \\
-1 \\
0 \\
0
\end{array}\right)=7+9=16 \\
& n_{4}^{\prime} \operatorname{sh}_{4}=(0,0,1,-1)\left(\begin{array}{rrrr}
12 & 5 & 8 & 2 \\
5 & 14 & 9 & 3 \\
8 & 9 & 15 & 4 \\
2 & 3 & 4 & 6
\end{array}\right)\left(\begin{array}{r}
0 \\
0 \\
1 \\
-1
\end{array}\right) \\
& =(8-2,9-3,15-4,4-6)\left(\begin{array}{c}
0 \\
0 \\
1 \\
-1
\end{array}\right)=(6,6,11,-2)\left(\begin{array}{c}
0 \\
0 \\
1 \\
-1
\end{array}\right)=11+2=13
\end{aligned}
$$

